\title{
Autostima e auto-compassione. Due modi diversi di relazionarsi con se stessi
}

di Emiliano Lambiase*, Bianca Elisa Lagioia**, Andrea Marino $^{\star * \star}$ e Noemi Grappone ${ }^{\star \star \star *}$

Il tema dell'autostima è ampiamente trattato in letteratura. Molti psicologi si sono interessati a questo argomento, in particolare perché il perseguimento di un buon livello di autostima è diventato una preoccupazione centrale nella cultura occidentale, in particolare in quella statunitense (Baumeister et al., 2003; Heine et al., 1999; Pyszczynski, Greenberg e Solomon, 1997; Sheldon et al., 2001). Centinaia di autori hanno svolto ricerche su questo argomento, sono stati pubblicati numerosissimi articoli negli ultimi 30 anni e l'interesse sembra continuare ad aumentare (Baumeister, 1998; Baumeister et al., 2003). Molte di queste ricerche hanno avuto come risvolto la stesura di manuali finalizzati a offrire ai genitori gli strumenti necessari per crescere figli con un buon livello di autostima (Benson, Galbraith e Espeland, 1998; Glennon, 1999; Miller, 2001). Numerose scuole hanno attuato programmi volti a rafforzare l'autostima degli studenti, nella speranza di ridurre seri problemi come l'alto tasso di abbandono scolastico, gravidanze in età adolescenziale e l'utilizzo di droghe e alcool (Dawes, 1994; McElherner e Lisovskis, 1998; Mecca, Smelser e Vasconcellos, 1989; Seligman, 1998).

Un ampio filone di ricerca, sostenuto da molti psicologi, considera che la ricerca dell'autostima sia così pervasiva in quanto bisogno umano universale e fondamentale (Allport, 1955; Baumeister, Heatherton e Tice, 1993; Maslow, 1968; Rogers, 1961; Rosenberg, 1979; Solomon, Greenberg e Pyszczynski, 1991; Taylor e Brown, 1988).

Di recente, tuttavia, altri ricercatori hanno contestato tali linee teoriche,

* Coordinatore Itituto di Terapia Cognitivo-Interpersonale.

** Ricercatrice Istituto di Terapia Cognitivo-Interpersonale.

*** Ricercatore Istituto di Terapia Cognitivo-Interpersonale.

**** Ricercatrice Istituto di Terapia Cognitivo-Interpersonale. 
sostenendo che i benefici oggettivi di un'alta autostima, in realtà, siano pochi e limitati. Ad esempio, una recente ed estesa rassegna svolta da Baumeister (Baumeister et al., 2003) ha concluso che l'alta autostima produce sensazioni piacevoli e maggiore iniziativa, ma non provoca alto rendimento scolastico, buone prestazioni lavorative o attitudine alla leadership.

Il presente articolo si pone l'obiettivo di svolgere una rassegna delle critiche mosse al costrutto dell'autostima e, in secondo luogo, di prestare attenzione ad una dimensione psicologica alternativa che si sta facendo sempre più strada nella letteratura scientifica: l'auto-compassione. La decisione di orientarsi in questa direzione è motivata dal fatto che in letteratura è presente un'attenzione sempre maggiore della psicologia verso le tradizioni spirituali. Infatti tematiche come l'accettazione, i valori, il perdono, la consapevolezza, la ricerca di significato, l'appartenenza e, appunto, la compassione sono sempre più oggetto di ricerca scientifica e di intervento clinico.

\section{Autostima}

Le teorie psicologiche sull'autostima si orientano in maniera unidirezionale sulla tesi che un basso livello di autostima è legato a una serie di esiti psicologici negativi quali ad esempio: mancanza di motivazione, depressione e ideazione suicidaria (vedi Harter, 1999, per una rassegna). Risulta invece meno chiaro come avvenga l'aumento di autostima e la sua ricerca: è infatti difficile accrescere l'autostima di un individuo, poiché è stato dimostrato come essa è altamente resistente al cambiamento (Swann, 1996).

Secondo Crocker e Park (2004), un problema centrale nelle ricerche sull'autostima è che non ci si è concentrati sufficientemente su ciò che fanno le persone per dimostrare a se stesse e agli altri che hanno dignità e valore $\mathrm{e}$ sulle conseguenze di tale ricerca. Per loro le persone perseguono l'autostima perché le aiuta a gestire le loro paure e ansie.

Stando a questa ipotesi, le situazioni e le azioni associate con l'autostima - e tutte le caratteristiche modalità con le quali la gente "lotta" per essa - si sviluppano durante l'infanzia. Infatti, è proprio in questo periodo della vita che gli esseri umani sono costitutivamente vulnerabili, richiedono cura e protezione dagli adulti e imparano le strategie per gestire la paura e cercare la rassicurazione (Bowlby, 1969, 1973).

Inevitabilmente i bambini sperimentano eventi che li minacciano, li spaventano o li sconvolgono. Da questi eventi, traggono alcune conclusioni su di sé ed elaborano considerazioni sugli altri, sul mondo e su come gestire eventi problematici futuri. A seguito di un evento sconvolgente, il bambino cerca di determinare che tipo di persona dovrà essere al fine di essere al si- 
curo e in modo che l'evento non si ripeta: ad esempio potrebbe ritenere di dover essere forte, autosufficiente o indipendente, così da non essere minacciato dall'abbandono; oppure, essere bello, affascinante, di successo o abbastanza ricco, in modo che non sarà ridicolizzato, respinto o criticato (Blatt, 1995). I settori in cui le persone scelgono di investire la loro autostima non sono necessariamente i domini in cui esse credono di riuscire quanto, piuttosto, i domini in cui, se dovessero avere successo, si sentirebbero al sicuro e protetti dai pericoli percepiti durante l'infanzia.

Purtroppo, nessun successo è in grado di garantire che eventi spiacevoli non accadano più. Nonostante il successo allevi momentaneamente l'ansia, essa tende a ripresentarsi. Pertanto, il perseguimento dell'autostima finalizzato alla soddisfazione delle contingenze di auto-efficacia continua inesorabilmente per tutto l'arco della vita.

Alcuni modi di perseguire l'autostima sembrano essere più dispendiosi al confronto di altri. Per esempio, le situazioni in cui la ricerca di validazione di sé dipende da conferme altrui hanno costi particolarmente rilevanti (Crocker, 2002; Pyszczynski, Greenberg e Goldenberg, 2002). Inoltre, in età adulta, l'autostima è costituita prevalentemente da giudizi e confronti con l'esterno (Coopersmith, 1967; Harter, 1999).

Come William James (1890) ha proposto più di un secolo fa, la stima di sé implica la valutazione delle prestazioni personali (quanto sono in gamba?), la determinazione di alcune norme (cosa è bene per me?) e la rilevanza percepita di tutto ciò in alcuni ambiti (è importante essere bravi in questa attività specifica).

L'autostima coinvolge, inoltre, la considerazione delle valutazioni degli altri su di sé (quanto e come gli altri si comportano con me e se mi approvano) al fine di determinare quanto ci si ama (Cooley, 1902, 1956; Mead, 1934). Il confronto sociale è quindi fattore determinante aggiuntivo nell'autostima (Aspinwall e Taylor, 1993; Beach e Tesser, 1995; Buunk, 1998; Deci e Ryan, 1995; Suls e Wills, 1991) in modo che il concetto di sé venga valutato in relazione alle prestazioni degli altri.

Il desiderio di validare l'autostima può distogliere l'attenzione delle persone dai propri obiettivi relativi alla ricerca di benessere e alla soddisfazione dei bisogni umani fondamentali (Crocker e Park, 2004; Vohs e Heatherton, 2001). In questo modo, alta e bassa autostima sono due facce della stessa medaglia, perché sia le persone con alta che quelle con bassa autostima comunque la perseguono, cercando di dimostrare di essere degni, non inutili e in entrambi i casi perdono di vista altri obiettivi.

Quando le persone cercano di conservare o migliorare l'autostima, le loro azioni sono guidate da convinzioni su ciò che devono fare o essere per avere dignità e valore (Dykman, 1998). Le persone differiscono su queste 
convinzioni e, quindi, su quali tipi di eventi produrranno una spinta o un calo nella loro autostima (Crocker e Wolfe, 2001). Il risultato dipende dal successo o dal fallimento percepito in questi domini specifici, che poi viene generalizzato al valore globale della persona (Crocker et al., 2003; Crocker, Sommers e Luhtanen, 2002).

In conclusione, si ritiene interessante che più autori sottolineano l'importanza di capire i motivi e le conseguenze della ricerca spasmodica di un aumento di autostima. Per questo motivo analizzeremo, divise in aree tematiche, le principali conseguenze negative di questa ricerca.

\subsection{Percezione di competenza e ottimismo}

La bassa autostima è correlata con una scarsa fiducia nelle proprie competenze e capacità. Inoltre, i soggetti con bassa autostima si presentano come persone pessimiste circa il loro futuro. Al contrario, chi ha un'alta autostima percepisce di essere efficiente, di avere buone competenze e capacità - talvolta anche esagerate - e ha aspettative ottimistiche verso il futuro (Alicke, 1985; Brown, 1986; Campbell, 1986; Taylor e Brown, 1988). Di conseguenza, ovviamente, le persone puntano ad avere un livello alto di autostima.

Effetto diretto del possesso di un alto livello di autostima è che la competenza percepita e l'ottimismo riducono l'ansia, veicolando la convinzione di essere in grado di gestire ciò che avviene nella vita: questo presupposto è direttamente collegato all'auto-efficacia (Franks e Marolla, 1976; Gecas e Schwalbe, 1983; Tafarodi e Swann, 1996, 2001).

Si può affermare, in realtà, che l'alta autostima è associata a un'illusione di competenza e ottimismo. Infatti, ci sono poche prove che il perseguimento di autostima permetta davvero di ottenere migliori risultati concreti (Baumeister et al., 2003; Colvin e Block, 1994). Ad esempio, gli studenti che basano la propria autostima su risultati accademici in realtà non ottengono voti superiori, anche se dichiarano di studiare di più (Crocker e Luhtanen, 2003).

$\mathrm{Si}$ qualifica quindi come uno sforzo da condurre con costanza per conservare la percezione di auto-efficacia e quindi tale da richiedere tempo ed energie pur non assicurando l'ottenimento, in modo proporzionale e diretto, dei risultati promessi. 


\subsection{Conseguenze motivazionali}

La ricerca dell'autostima è legata al raggiungimento di svariati vantaggi, ad esempio: successo professionale o finanziario, realizzazione affettiva, fama. Un dibattito di lungo periodo, nella letteratura di auto-aiuto, muove intorno la seguente quaestio: l'offerta di aiuto nasce da motivazioni altruistiche o è guidata da intenzioni egoistiche (cf. Batson, 1987, 1998)?

La ricerca di autostima, quindi, per molte persone potrebbe essere un forte motore motivazionale. Tuttavia sono poche le prove che la motivazione associata al perseguimento di autostima si traduca realmente nel successo desiderato. Invece, ce ne sono molte che quando l'obiettivo centrale è proteggere, mantenere e potenziare l'autostima, le persone divengono suscettibili a stress, pressione, ansia per il possibile fallimento (Deci e Ryan, 1995; Ryan, 1982). Ad esempio, gli studenti che basano la propria autostima sui risultati accademici riportano più pressione, più conflitti con i professori, più insoddisfazione per le loro prestazioni e una motivazione meno intrinseca indipendentemente dal livello di autostima, dalla media dei voti e dalle variabili di personalità (Crocker e Luhtanen, 2003; Deci, Nezlek e Sheinman, 1981; Deci e Ryan, 2000; Ryan e Deci, 2000). In realtà, il perseguimento dell'autostima può interferire con l'apprendimento e le prestazioni in circostanze difficili o impegnative, infatti, potrebbe causare scarsa autodisciplina, minare l'autonomia personale e le relazioni.

Inoltre, la ricerca indica che in settori in cui l'autostima è centrale, sono maggiori i casi in cui i soggetti subiscono un elevato calo di autostima successivamente a un fallimento e rispetto a quanto questa aumenti come risultato di un successo (Crocker et al., 2003; Crocker, Sommers e Luhtanen, 2002). Così, i benefici emotivi del perseguimento di autostima superano i costi solo se le persone sono in grado di garantire di poter riuscire di più rispetto a quanto possono fallire. Per le persone, quindi, la ricerca di autostima è un fattore motivazionale importante nelle loro varie attività, ma è anche altamente volatile per lo stress che produce e perché deriva costantemente la sua forza dalle conferme esterne e non dall'interno della persona. Inoltre, senza tale motivazione, queste persone sarebbero totalmente prive di obiettivi o di ambizione. D'altro canto, anche se la ricerca di autostima è in grado di fornire una fonte importante di motivazione, altre motivazioni potrebbero guidare lo stesso comportamento. Ad esempio, gli studenti possono voler fare bene a scuola perché a) saranno ricompensati; b) si sentiranno bene con se stessi; c) credono sia importante; o d) gli piace (Ryan e Connell, 1989). Così, rinunciare alla ricerca di autostima non porta a una mancanza di motivazione. 


\subsection{Autonomia}

Una conseguenza diretta delle dinamiche a livello motivazionale appena descritte è la perdita di autonomia nella scelta dei propri obiettivi. L'autonomia si riferisce alla sensazione di essere in grado di volere, scegliere e causare il proprio comportamento (deCharms, 1968; Deci e Ryan, 2000).

Come suggeriscono Deci e i suoi colleghi (Deci et al., 1994), quando subordiniamo la nostra autostima al raggiungimento di un risultato lasciamo che sia una situazione esterna a regolare il nostro stato interno. Così, arriviamo a comportarci in un certo modo perché sentiamo di doverci comportare in quel modo e non perché lo vogliamo; questa modalità di auto-regolazione è accompagnata dall'esperienza interiore di pressione e di tensione. In questo senso, quindi, perdiamo autonomia nella scelta degli obiettivi. Quando le persone cercano di proteggere, mantenere e migliorare la loro autostima, perdono la capacità di agire in modo autonomo perché orientano la loro attenzione e le loro energie verso quegli obiettivi rilevanti per l'autostima e parallelamente aumentano lo stato di stress interno riducendo anche le proprie risorse fisiche e mentali a causa degli stati fisici e mentali conseguenti. In questo senso, quindi, si perde anche quella forma di autonomia che consiste nel sentirsi liberi interiormente, anche quando si è affaticati nel raggiungimento di propri obiettivi. In sintesi, questi risultati suggeriscono che quando le persone cercano di proteggere, mantenere e migliorare la loro autostima, perdono la capacità di agire in modo autonomo: sia nella determinazione degli obiettivi, che nel modo in cui tali obiettivi vengono vissuti interiormente.

\subsection{Apprendimento e competenza}

Competenza e apprendimento sono fra i bisogni principali dell'essere umano (Bowlby, 1969; Deci e Ryan, 2000; White, 1959). Nelle trattazioni che sostengono questo punto di vista, la competenza non si riferisce al contenuto della propria conoscenza o al livello delle proprie abilità quanto, piuttosto, alla capacità e alla volontà di imparare e di crescere nell'esperienza (vedere Pyszczynski, Greenberg e Goldenberg, 2002 per una discussione).

Il perseguimento di un alto livello di autostima, però, si è visto che interferisce con l'apprendimento e la competenza (Covington, 1984; Deci e Ryan, 2000; Dweck, 2000).

Quando le persone hanno obiettivi di auto-validazione, vivono gli errori, i fallimenti e le critiche come minacce e non come opportunità per imparare e migliorare. Nei domini in cui viene investita l'autostima, diviene fondamentale ottenere risultati. L'apprendimento, che dovrebbe avere lo scopo di 
arricchirci come persone, diventa solo un mezzo per ottenere delle performance, perdendo così il suo vero fine.

Di conseguenza, quando le persone sono guidate da obiettivi collegati all'autostima, sono desiderose di sperimentare il merito del loro successo (Blaine e Crocker, 1993; Bradley, 1978; Greenberg e Pyszczynski, 1985; Miller e Ross, 1975). Nel momento in cui l'alta autostima diventa l'obiettivo centrale, coloro che riescono a ottenere dei buoni risultati possono automaticamente percepire di aver soddisfatto il loro bisogno. Ciò implica, però, che questi stessi soggetti non riescono a compiere una disamina completa dei fattori che hanno contribuito al loro successo: per esempio, non riescono ad analizzare lucidamente il contesto, gli sforzi di altre persone, il cambiamento delle circostanze e così via (Carver, 2003). Come si può facilmente intuire, questo processo può interferire con l'apprendimento.

Tali persone, nell'esperienza di orgoglio che accompagna il successo, possono anche arrivare a immaginare o desiderare maggiori successi (Fredrickson e Branigan, 2001). Nel momento in cui l'obiettivo non viene raggiunto, invece, possono reagire in modo difensivo alle informazioni negative su di sé, relative alle aree vissute come importanti per l'autostima: ad esempio, possono mettere in discussione tali informazioni (Baumeister, 1998); mettere in atto una serie di giustificazioni (Blaine e Crocker, 1993; Bradley, 1978; Greenberg e Pyszczynski, 1985; Miller e Ross, 1975; Kernis e Waschull, 1995); minimizzare la quantità di tempo e le energie investite a pensare alle informazioni negative che riguardano se stessi (Baumeister e Cairns, 1992); dimenticare fallimenti e informazioni negative su di sé, ricordando solo successi e informazioni positive (Crary, 1966; Mischel, Ebbesen e Zeiss, 1976); cercare gratificazioni compensatorie (es. Baumeister e Jones, 1978; Gollwitzer e Wicklund, 1985; Greenberg e Pyszczynski, 1985); confrontarsi con altre persone più deboli (Beauregard e Dunning, 1998; Crocker et al., 1987; Pyszczynski, Greenberg e Laprelle, 1985; Wills, 1981; Wood, Giordano-Beech e Ducharme, 1999); ricordare informazioni negative sugli altri (Crocker, 1993); prendere le distanze da chi ritengono li sovrastimi (Tesser, 1988, 2000); incolpare gli altri (Aberson, Healy e Romero, 2000; Crocker e Luhtanen, 1990; Crocker et al, 1987; Fein e Spencer, 1997; Spencer et al., 1998; Wills, 1981); barare (Covington, 1984, 2000; Dweck, 2000).

Quando il fallimento o la critica non possono essere giustificati, questi possono essere generalizzati fino al punto di diventare un atto d'accusa verso di sé, abbassando globalmente l'autostima (Carver e Ganellen, 1983; Carver et al., 1988; Crocker et al., 2003; Crocker, Sommers e Luhtanen, 2002). Inoltre, auto-sabotarsi è un ottimo esempio di creazione di barriere 
per il proprio successo al fine di proteggere o migliorare la stima di sé attraverso la creazione di una scusa per il proprio fallimento, non dovendo arrivare a toccarlo davvero con mano (Tice, 1991).

Risulta evidente come tutte queste modalità minano alla radice la possibilità di apprendere e sviluppare le proprie competenze.

\subsection{Costi per le relazioni}

Le relazioni intime forniscono cura e sostegno. Diventano come un "porto sicuro" in momenti di difficoltà (Baumeister e Leary, 1995; Collins e Feeney, 2000; Deci e Ryan, 2000), contribuiscono a creare strategie più efficaci per affrontare momenti critici (Cohen, Sherrod e Clark, 1986), favoriscono una migliore longevità mentale e in generale una migliore salute fisica (Ryff, 1995). Questo fa capire perché alcune persone danno molta importanza al perseguimento dell'autostima, dato che quest'ultima fornisce la percezione di essere accettati socialmente, presumibilmente perché aumenta la percezione del proprio valore relazionale.

Le persone perseguono l'autostima perché quando hanno successo si sentono sicure che gli altri le accoglieranno e la loro ansia di essere esclusi diminuisce. Tuttavia, esistono poche prove che le cose che la gente fa per aumentare l'autostima e quindi la percezione di inclusione sociale, aumentino realmente la popolarità, le connessioni interpersonali e/o i rapporti di sostegno reciproco. Ad esempio persone con alta autostima incolpano gli altri a seguito di minacce auto-inflitte; questo comportamento protegge l'autostima, ma li fa apparire antagonisti e/o antipatici agli altri (Heatherton e Vohs, 2000).

Dalle ricerche emerge che le persone che si focalizzano sull'autostima sono meno sintonia con i bisogni e i sentimenti altrui (Park e Crocker, 2003) e desiderano essere superiori agli altri (Brown, 1986; Taylor e Brown, 1988). Di conseguenza, la vita si trasforma in un uno scambio fondato sull'equilibrio tra dare e avere, per cui le altre persone diventano concorrenti se non nemici, piuttosto che sostegni e risorse. Le relazioni verranno viste come una minaccia continua a cui rispondere con evitamento, allontanamento, fuga, colpa, scuse, rabbia, antagonismo e aggressività (Baumeister, 1998; Baumeister, Bushman e Campbell, 2000; Baumeister, Smart e Boden, 1996; Crocker e Park, 2003; Heatherton e Vohs, 2000; Kernis e Waschull, 1995; Tice, 1993). Queste reazioni difensive possono causare isolamento e distacco dagli altri e ostacolare la formazione di significativi e autentici rapporti di sostegno (Pyszczynski, Greenberg e Goldenberg, 2002).

Il desiderio di un'alta autostima, inoltre, può comportare la volontà di 
vedere il peggio negli altri come mezzo per incrementare la valutazione di sé (Crocker et al., 1987; Feather, 1994). In effetti, l'alta autostima, piuttosto che quella bassa, è stata associata ad un aumento del pregiudizio verso $\mathrm{i}$ gruppi esterni rispetto a quello di appartenenza (Aberson, Healy e Romero, 2000; Allport, 1954; Turner et al., 1987).

Le persone con bassa autostima tendono a focalizzarsi sull'essere amate, incluse o accettate (Joiner, Katz e Lew, 1999; Joiner et al., 1999; Vohs e Heatherton, 2001) e rispondono alle minacce al concetto di sé cercando rassicurazione dagli altri (Murray et al., 2001). Tale desiderio di approvazione e rassicurazione esterna produce un'eccessiva sensibilità ai segni reali $\mathrm{o}$ immaginari di rifiuto, la supposizione che il comportamento negativo (percepito o reale) degli "altri significativi" rifletta intenzioni ostili e la messa in atto di azioni di difesa che alla fine minano il rapporto (Murray et al., 2001).

In particolare, gli uomini con sensibilità al rifiuto tendono a reagire con gelosia, ostilità e tentativi di controllare il partner, mentre le donne tendono a ritirare il sostegno e a diventare scoraggiate (Downey e Feldman, 1996). Nel corso del tempo, questo stile di interazione può ostacolare il sostenersi a vicenda e infine portare alla dissoluzione dei rapporti (Downey et al., 1998). Pertanto, le persone che cercano l'approvazione degli altri (e contemporaneamente temono il rifiuto) creano esattamente il rifiuto paventato (Downey et al., 1998; Joiner, 1994; Joiner, Alfano e Metalsky, 1992; Joiner, Metalsky et al., 1999; Murray, Holmes e Griffin, 2000; Downey et al., 1998).

Crocker e Park (2004), inoltre, suggeriscono che la preoccupazione per le implicazioni che determinate azioni possono avere per il concetto di sé, induce la gente a perdere di vista le implicazioni degli eventi e delle proprie azioni sugli altri. Queste persone utilizzano meno risorse cognitive per comprendere il punto di vista dell'altro e, quindi, non riescono a prendere in considerazione ciò di cui gli altri hanno bisogno o che cosa è bene per loro. Di conseguenza, gli altri sperimentano sentimenti di diffidenza e insicurezza. Inoltre, come abbiamo già detto, l'obiettivo di convalidare l'autostima crea spesso competizione e il desiderio di essere superiori agli altri. Questo, a sua volta, fa scattare la competizione negli altri che non vogliono essere da meno e può creare il desiderio di vendetta o ritorsione.

\subsection{Autoregolazione}

L'autoregolazione comporta la capacità di un individuo di poter controllare quei comportamenti per i quali conosce le conseguenze negative indesiderate, così come la capacità di perseguire gli obiettivi dai quali ritiene di poter ricevere benefici futuri (Metcalfe e Mischel, 1999). Le abilità di auto- 
regolazione e autocontrollo hanno dimostrato benefici a lungo termine (Mischel e Shoda, 1995; Shoda, Mischel e Peake, 1990). Come Baumeister (1998) fa notare, un'elevata capacità di autoregolazione sembra essere un bene assoluto che migliora le probabilità di successo in quasi ogni sforzo rilevante.

Per quanto concerne la relazione tra autostima e auto-regolazione, le persone che sono focalizzate sul migliorare o difendere il concetto di sé hanno difficoltà ad autoregolare il proprio comportamento.

Metcalfe e Mischel (1999) hanno sostenuto che ci sono due sistemi per l'autocontrollo e l'auto-regolazione: uno "freddo" e cognitivo, uno "caldo" ed emotivo. Il primo permette di rimanere focalizzati sui propri obiettivi monitorando i progressi lungo il percorso per il loro raggiungimento. Il secondo, invece, è veloce, immediato, enfatizzato dallo stress e strettamente collegato agli stimoli attivanti.

Poiché l'autostima ha conseguenze importanti sull'emotività, il suo perseguimento è in gran parte sotto il controllo del sistema "caldo". Nel momento in cui il concetto di sé è minacciato, spesso si indulge in impulsi immediati allo scopo di sentirsi meglio, dando la priorità alla regolazione a breve termine rispetto ad altri obiettivi di auto-regolazione (Tesser, 1988; Tice e Bratslavsky, 2000; Tice, Bratslavsky e Baumeister, 2001).

Come già detto, quando le persone hanno obiettivi inerenti l'autostima sono motivate a vedere se stesse in una luce positiva, deviando la responsabilità per il fallimento e cercando, piuttosto, di ascriversi il merito per il successo. Come risultato, l'auto-regolazione ne risente perché il soggetto si trova in difficoltà nel valutare realisticamente la realtà effettuale e confrontarla con lo stato ideale; si può dunque diventare eccessivamente positivi o negativi rispetto a eventuali discrepanze o evitare del tutto di focalizzarsi sulle mancanze altrui. Inoltre, si possono incontrare difficoltà nel valutare $\mathrm{i}$ progressi verso il raggiungimento di un obiettivo (Wilson e Ross, 2000, 2001). Si ha un'efficace auto-regolazione nel momento in cui si riesce a svincolarsi dagli obiettivi quando i progressi sono troppo lenti (Carver e Scheier, 1998), ma spesso si ha difficoltà a svincolarsi dagli obiettivi connessi all'autostima (Baumeister, Heatherton e Tice, 1993; Pyszczynski e Greenberg, 1987).

Crocker e Park (2004), sottolineano che il perseguimento dell'autostima può portare all'esaurimento delle risorse di auto-regolazione in quanto vengono dissipate dallo sforzo richiesto per soddisfare le richieste del sistema "a caldo". Quando si verifica ciò, non si è in grado di esercitare l'autocontrollo anche in altri settori in cui le persone solitamente si autoregolano con successo.

L'uso del tempo (Crocker e Park, 2004) è un altro aspetto importante dell'auto-regolazione. Nella ricerca di autostima il tempo viene utilizzato per 
preoccuparsi, procrastinare, auto-sabotarsi e ricercare la perfezione. Quello che viene a mancare è il senso di accettazione del limite e dell'imperfezione. Viene ad esaurirsi, in questo modo, il tempo che potrebbe essere usato per attività che probabilmente avrebbero maggiori probabilità di farci raggiungere degli obiettivi e soddisfare i bisogni umani fondamentali.

\subsection{Costi per la salute fisica}

Gli obiettivi connessi all'autostima possono portare a problemi di salute fisica attraverso l'ansia e lo stress che producono (Suinn, 2001). Persone con alti obiettivi di autostima tendono ad essere molto ansiose (Dykman, 1998) e l'ansia ha effetti negativi sulla salute (Suinn, 2001). Una breve panoramica teorica lascia emerge che stress e ansia sono associati con l'attivazione del sistema ipofisi-surrene-corticale che rilascia corticosteroidi dalla corteccia surrenale (Hellhammer e Wade, 1993; Stroebe, 2000). I corticosteroidi aumentano i livelli di trigliceridi e di colesterolo nel sangue, cosicché lo stress cronico è spesso associato a malattie cardiache. I corticosteroidi riducono anche il funzionamento del sistema immunitario (KiecoltGlaser et al., 1992; Kiecolt-Glaser e Glaser, 1994), con conseguente maggiore suscettibilità alle malattie come ad esempio le infezioni delle vie respiratorie superiori (Kiecolt-Glaser et al., 1991). Lo stress è associato anche con l'attivazione del sistema simpatico-midollare-surrenale, che stimola l'attività cardiovascolare con conseguenze per la salute cardiaca (Stroebe, 2000; Suinn, 2001). Non sorprende, dunque, per fare un esempio, che gli studenti che procrastinano gli esami si ammalano più frequentemente e riportano più stress e malattie nel lungo periodo (Tice e Baumeister, 1997).

Si può pertanto ipotizzare, che siccome stress e ansia possono essere diretta conseguenza di una ricerca intensa di autostima, quest'ultima possa provocare, o essere una concausa, di alcune sintomatologie sopra descritte. Infine, per far fronte all'effetto negativo associato con il perseguimento dell'autostima, le persone spesso iniziano a bere alcolici o ad avere rapporti sessuali non protetti, con conseguenze potenzialmente gravi per la salute (Cooper, Agocha e Sheldon, 2000; Cooper et al., 1995; Cooper, Shapiro e Powers, 1998; Hull, 1981; Hull et al., 1983; Hull e Young, 1983; Stroebe, 2000; Suinn, 2001; Cantelmi e Lambiase, 2007). 


\subsection{L'impatto emotivo e sulla salute mentale}

Negli ultimi anni, diverse critiche sono state fatte all'uso dell'autostima come misura primaria della salute psicologica (Baumeister, Smart e Boden, 1996; Damon, 1995; Ellis e London, 1993; Finn, 1990; Hewitt, 1998, McMillan, Singh e Simonetta, 1994; Seligman, 1995; Swann, 1996) e, anzi, il perseguimento di un buon concetto di sé sembra avere implicazioni per la salute mentale, in particolare depressione, narcisismo e ansia (Crocker e Park, 2004).

Secondo Crocker e Nuer (2004) la ricerca non ha dimostrato che le persone hanno bisogno di autostima, che perseguire l'autostima sia un mezzo efficace per ridurre l'ansia e che avere o perseguire un'elevata autostima aiuti a raggiungere i propri obiettivi. Anche se una bassa autostima è correlata con la presenza di sintomi depressivi, la prova che una bassa autostima sia un fattore di rischio per la depressione, piuttosto che un sintomo di essa, non è definitiva (Roberts e Gamble, 2001; Tennen e Herzberger, 1987).

Secondo Crocker e Park (2004), è il perseguimento dell'autostima a essere un fattore di rischio per lo sviluppo della depressione. Infatti, le persone che tendono ad affrontare le situazioni e gli eventi di vita con obiettivi collegati all'autostima hanno molteplici sintomi depressivi (Dykman, 1998). La tendenza a generalizzare gli eventi negativi rispetto al proprio valore, che è una caratteristica delle persone con obiettivi collegati all'autostima, è legata alla depressione e predice lo sviluppo, in prospettiva, di sintomi depressivi (Carver, 1998; Carver e Ganellen, 1983; Carver et al., 1988). Inoltre, l'instabilità dell'autostima causata dal successo e dal fallimento in settori percepiti come importanti può contribuire allo sviluppo dei sintomi depressivi (Butler, Hokanson e Flynn, 1994; Kernis et al., 1998; Kuiper e Olinger, 1986; Kuiper, Olinger e MacDonald, 1988; Roberts e Gotlib, 1997; Roberts, Kassel e Gotlib, 1995, 1996; Roberts e Monroe, 1992).

Il successo nella ricerca di autostima produce un temporaneo aumento di emozioni positive, fra cui l'orgoglio (Crocker et al., 2003; Crocker, Sommers e Luhtanen, 2002; Lewis, 1993; Mascolo e Fischer, 1995). Al contrario, il fallimento porta a cali di quest'ultima e all'aumento di tristezza, rabbia, vergogna e di altre emozioni negative intense (Tangney, 1999).

Dykman (1998) ha rilevato che le persone che si focalizzano solo su obiettivi di auto-validazione sperimentano ansia. Avere alti livelli di autostima riduce questa ansia ma, d'altro canto, l'obiettivo di convalidare l'autostima evitando eventuali dimostrazioni della propria inutilità è collegato a maggiore ansia. In sintesi, anche se si aumenta l'autostima per ridurre l'ansia, il perseguimento di autostima può aumentarla. 
Quando le persone raggiungono il successo nei settori in cui la loro autostima è messa in gioco, sperimentano un incremento nella stima di sé, un aumento delle emozioni positive e una diminuzione di sensazioni negative tra cui l'ansia (Crocker et al., 2003; Crocker, Sommers e Luhtanen, 2002). Tale aumento, però, è temporaneo. Ad esempio, anche se gli studenti più anziani del college che basano la propria autostima su risultati accademici mostrano un livello più alto della stessa e una riduzione degli effetti negativi quando sono ammessi a un corso di laurea, la spinta dell'autostima dura al massimo un paio di giorni per poi ritornare al suo valore di base (Crocker et al., 2002). Crocker e Nuer (2003) paragonano il perseguimento dell'autostima al tentativo di sfuggire all'ansia tramite l'assunzione di alcool o farmaci: diventa implacabile, l'ansia ritorna sempre rendendo necessario un ulteriore incremento di autostima, in una rincorsa continua di successi e risultati da raggiungere. I tentativi diretti di aumentare l'autostima possono portare $\mathrm{i}$ giovani a diventare più narcisisti o antisociali (Baumeister, Smart e Boden, 1996) e ad aggrapparsi a concetti positivi di sé ed evitare, di conseguenza, opportunità impegnative di apprendimento che potrebbero minacciare tale concetto (Dweck, Chiu e Hong, 1995; Mueller e Dweck, 1998). Infine, la ricerca di stima di sé può essere collegata a rabbia o aggressività verso le proprie debolezze e alla mancanza di empatia (Seligman, 1995).

\section{L'auto-compassione}

La compassione implica l'essere toccati dalla sofferenza e dal dolore altrui, così che emergano sentimenti di gentilezza verso gli altri e il desiderio di alleviare le loro sofferenze (Wispe, 1991). La compassione, inoltre, implica l'offerta di comprensione non giudicante verso coloro che sbagliano, in modo che le loro azioni vengano viste nel contesto della fallibilità umana condivisa. Di conseguenza, l'auto-compassione implica l'essere toccati dalla propria sofferenza, senza evitarla o cercando di allontanarsi da essa, ma desiderando di alleviare il proprio dolore e di guarire se stessi con gentilezza.

L'auto-compassione comporta l'offrire comprensione non giudicante ai propri dolori, carenze e fallimenti, riconoscendo che questi fanno parte della condizione umana e che tutte le persone, inclusi noi, siamo degni di compassione. Questo atteggiamento tenderà a rafforzare anche i sentimenti di compassione e preoccupazione verso gli altri.

Così, provare compassione per se stessi è simile a perdonarsi. Enright osserva che quando perdoniamo, «accogliamo con favore l'altro nella comunità umana e ci vediamo come altrettanto degni di rispetto» (Enright, 
Freedman e Rique, 1998, p. 49). Allo stesso modo, l'auto-compassione comporta indulgenza rispetto ai propri difetti, rispettando se stessi come esseri umani e quindi limitati e imperfetti.

Sebbene avere auto-compassione richieda di non criticare aspramente se stessi per non aver rispettato i propri standard ideali, ciò non significa che le proprie mancanze passino inosservate o che non vengano corrette. Piuttosto, le azioni necessarie per il funzionamento ottimale e per la salute vengono attuate con dolcezza e pazienza. È la mancanza di auto-compassione, piuttosto, a portare alla passività dato che, quando ci si sente severamente giudicati per le proprie mancanze, nella convinzione che l'auto-flagellazione in qualche modo possa forzare il cambiamento e il miglioramento di sé, la mente agisce in modo da nascondere alla consapevolezza le inadeguatezze cosicché l'autostima non sia in pericolo (Horney, 1950; Reich, 1949). Senza la consapevolezza di sé, queste debolezze rimarranno incontrastate. Attraverso la compassione per se stessi, quindi, si fornisce la sicurezza emotiva necessaria per analizzarsi e comprendersi chiaramente, senza timore dell'auto-condanna, dando a se stessi il permesso di percepirsi e comprendersi in modo più accurato e di correggere schemi mentali disadattivi (Brown, 1999). Inoltre, la cura di sé, intrinseca all'auto-compassione, dovrebbe fornire un potente stimolo per la crescita e il cambiamento.

Leary e colleghi (2007) hanno intrapreso una serie di esperimenti per esaminare i processi cognitivi ed emotivi con cui le persone con autocompassione gestiscono gli eventi di vita negativi. Il gruppo di ricerca ha scoperto che l'auto-compassione agisce come un cuscinetto contro le emozioni negative nel momento in cui i partecipanti alla ricerca erano impegnati in un evento interpersonale che coinvolgeva un'autovalutazione sfavorevole o negativa.

Va inoltre notato che l'auto-compassione è ben distinta dall'autocommiserazione (Goldstein e Kornfield, 1987). Quando gli individui sentono pietà per gli altri, in genere si sentono altamente indipendenti e scollegati da loro («grazie a Dio è un problema tuo e non mio»), immergendosi nei propri problemi senza curarsi di quelli altrui. Al contrario, gli individui compassionevoli si percepiscono legati agli altri e sono consapevoli che la sofferenza è qualcosa che tutti gli esseri umani esperiscono. L'autocommiserazione tende a sottolineare i sentimenti egocentrici di separazione dagli altri e ad esagerare la portata della sofferenza personale. L'auto-compassione, invece, permette di vedere le esperienze personali e altrui senza questo tipo di distorsione o disconnessione.

Un altro modo in cui l'auto-compassione è distinta dall'auto-commiserazione riguarda la misura in cui gli individui si identificano con il dolore e la sofferenza personali. Le persone che vivono nell'autocommiserazione si sentono 
completamente assorbite dai loro sentimenti. Questo processo può essere definito come "sovra-identificazione" e si manifesta nel momento in cui gli individui diventano così immersi nelle loro attuali reazioni emotive che altri aspetti della persona, quelli in grado di fornire risposte emotive alternative, diventano inaccessibili (Goleman, 1995; Bennett-Goleman, 2001). Nella "sovra-identificazione" la consapevolezza è completamente oscurata dalle reazioni soggettive e non si riesce ad adottare una prospettiva obiettiva. Nel caso di emozioni negative associate a fallimento o a inadeguatezze personali, c'è un focus esagerato sulle implicazioni per l'autostima, che conduce a giudizi eccessivamente severi e a critiche su di sé. La "sovra- identificazione" incrementa i sentimenti di separazione e di isolamento, amplifica il senso di sé e oscura la consapevolezza che tutti gli esseri umani esperiscano sofferenza e delusione. Purtroppo, la ruminazione, l'autocritica e i sentimenti di separazione hanno dimostrato di essere altamente associati a esiti disadattivi come la depressione (Blatt et al., 1982; Bowlby, 1980; Nolen-Hoeksema, 1991).

Al contrario, l'auto-compassione richiede che gli individui non si "sovra-identifichino" con le loro emozioni, in modo che ci sia uno "spazio mentale" in cui espandere se stessi con gentilezza e riconoscere il contesto umano più ampio della propria esperienza (Goldstein e Michaels, 1985; Scheff, 1981). L'auto-compassione è meno dipendente da circostanze esterne e si concentra più sulla valorizzazione di sé, pur riconoscendo le imperfezioni soggettive (Neff, 2011). Come convalida interna al concetto di sé, l'auto-compassione consiste in un meccanismo di coping che porta benefici a se stessi e agli altri durante i periodi di tristezza e delusione (Baker e McNulty, 2011; Neff e Vonk, 2009).

In sintesi, secondo Neff (2003), di fronte ad esperienze di sofferenza o di fallimento personale, l'auto-compassione consta di tre componenti di base: (a) la gentilezza e la comprensione verso se stessi, al posto di un giudizio e una critica duri verso di sé; (b) la capacità di guardare alle proprie esperienze come parte dell'esperienza umana condivisa, piuttosto che vederle come separate e isolate; e, infine, (c) la capacità di gestire i propri pensieri e sentimenti dolorosi in uno stato di consapevolezza equilibrata, piuttosto che identificarsi eccessivamente con loro. Questi aspetti di autocompassione sono concettualmente distinti e sono esperiti in maniera differente a livello fenomenologico, ma interagiscono reciprocamente al fine di migliorare l'un l'altro.

\section{Autostima e auto-compassione: due costrutti a confronto}

Nei paragrafi che seguono ci si propone di compiere un excursus teorico 
che metta a confronto i costrutti di autostima e compassione, evidenziandone le caratteristiche in comune e le differenze.

\subsection{Compassione e relazioni}

La compassione gioca un ruolo importante non solo nel modo con il quale ci relazioniamo con noi stessi quando siamo in rapporto solo con noi stessi (ad es. come mi tratto per un mio errore), ma anche nel modo con il quale ci confrontiamo con gli altri nei rapporti interpersonali (come mi tratto quando sto in relazione con l'altro, oppure come tratto l'altro quando si relaziona con me). Se nel corso dell'articolo abbiamo per lo più focalizzato l'attenzione sulla compassione verso se stessi nella relazione con se stessi, ora ci focalizzeremo in modo specifico sulle ricerche che hanno messo in evidenza l'importanza della compassione verso se stessi o verso l'altro nella relazione interpersonale.

\subsubsection{Compassione per l'altro nella relazione con l'altro}

Un numero sempre crescente di ricerche suggerisce $\mathrm{i}$ benefici non intenzionali nell'essere responsivi e nel sostenere gli altri (Brown et al., 2003; Crocker et al., 2010; Deci et al., 2006; Aknin, Dunn e Norton, 2009; Piferi e Lawler, 2006).

Gli obiettivi che hanno come scopo costruire, mantenere e difendere l'immagine di sé creano dinamiche interpersonali negative che minano i rapporti intimi e la salute mentale. Al contrario, gli obiettivi compassionevoli che hanno come scopo sostenere il benessere altrui, creano dinamiche interpersonali positive che promuovono le relazioni intime e la salute mentale.

Una grande mole di evidenze scientifiche suggerisce che i rapporti interpersonali di alta qualità allontanano le persone dal malessere (Berkman et al., 2000; Cohen e Wills, 1985; House, Landis e Umberson, 1988; Uchino, 2004). Quando le persone non hanno rapporti intimi o quando la qualità delle loro relazioni è scarsa ne risente persino la loro salute fisica (Ali et al., 2006; Hawkley e Cacioppo, 2003; Kiecolt-Glaser et al., 2003).

Avere come centro del proprio interesse se stessi, spesso può portare ad allontanarsi dagli obiettivi sociali. Canevello e Crocker (2011a) considerano questa possibilità distinguendo tra due categorie di obiettivi ed esaminando le conseguenze per sé e gli altri: gli obiettivi che si concentrano sul sé, sulle esigenze proprie e sul proprio benessere e gli obiettivi compassionevoli. 
Quando le persone hanno come obiettivo la propria immagine personale, si concentreranno su cosa possono guadagnare dall'apparire agli occhi degli altri in un determinato modo, cercando di controllare questo tipo di risultato (cf. Baumeister, 1982 per una rassegna; Jones e Pittman, 1982; Leary, 1995; Leary e Kowalski, 1990; Schlenker, 1980; Tedeschi, 1981). In questo modo, però, non si può aver cura degli altri o dei loro bisogni (Gollwitzer e Wicklund, 1985).

Nella seconda categoria di obiettivi, invece, le persone ampliano i propri ambiti e li estendono oltre se stessi concentrandosi sui bisogni e sul benessere degli altri con autentica cura o preoccupazione (Batson, 1998; Clark e Mills, 1993; Davis, 1983; Feeney e Collins, 2003; Fehr e Sprecher, 2009; Helgeson, 1994). Canevello e Crocker (2011a) sostengono che quando le persone hanno obiettivi compassionevoli e sostengono il benessere degli altri, creano dinamiche relazionali e contesti che favoriscono relazioni positive e promuovono la salute mentale, garantendo anche un paracolpi rispetto al malessere. Tali autori, comunque, evidenziano come le due categorie di obiettivi non si escludono a vicenda, potendo quindi essere perseguite contemporaneamente (Canevello e Crocker, 2010; Crocker e Canevello, 2008).

La letteratura scientifica identifica la responsività come un principio fondamentale nelle relazioni intime (Clark e Lemay, 2010; Reis, Clark e Holmes, 2004) e lo definisce come il processo attraverso cui gli altri «si occupano e danno supporto all'essenza delle caratteristiche del sé» (Reis, Clark e Holmes, 2004, p. 203). Quando le persone credono che nelle relazioni, i partner siano responsivi, si sentono compresi, validati e curati (Gable e Reis, 2006), il che li porta a sentirsi intimi, appagati e impegnati in quei rapporti (Canevello e Crocker, 2010; Reis, Clark e Holmes, 2004).

Canevello e Crocker (2011a) ritengono che l'immagine di sé e gli obiettivi compassionevoli influenzino la percezione che le persone hanno riguardo la responsività del loro partner, la qualità del rapporto e le mete future.

Le persone si pongono obiettivi relativi all'immagine di sé quando credono che gestendo le impressioni che gli altri hanno di loro stessi, possono ottenere ciò che vogliono o di cui hanno bisogno (Schlenker, 2003) ed essendo focalizzate su questi obiettivi, rischiano di essere meno sensibili verso il partner. Questa dinamica, successivamente, li conduce a percepire anche il partner come meno sensibile nei loro confronti (Lemay e Clark, 2008; Lemay, Clark e Feeney, 2007) e, come risultato, emerge un rapporto di bassa qualità (Reis, Clark e Holmes, 2004). Di converso, quando si ha l'obiettivo compassionevole di sostenere il benessere degli altri, si dovrebbe essere più sensibili al partner e percepirlo anche come più responsivo, migliorando di conseguenza anche la qualità della relazione. Percepire $\mathrm{i}$ 
partner come sensibili favorisce la sicurezza del rapporto, il che può consentire uno spostamento dell'attenzione dalla protezione di sé all'essere di supporto per gli altri (Mikulincer e Shaver, 2005; Murray, Holmes e Collins, 2006), stimolando così l'assunzione di obiettivi compassionevoli.

Inoltre, le persone notano la mancanza di responsività degli altri e, come conseguenza, li trattano a loro volta in modo meno responsivo (Canevello e Crocker, 2010). Questa mancanza di responsività crea una distanza nelle relazioni che ostacola la percezione di sicurezza e soddisfazione della relazione, il senso di vicinanza e di impegno (es. Canevello e Crocker, 2010; Reis, Clark e Holmes, 2004).

Sembra quindi ragionevole ipotizzare che una minor responsività associata agli obiettivi relativi all'immagine di sé, possa predire una diminuzione della considerazione degli altri verso di sé.

Una bassa responsività può portare a un calo dell'autostima perché quando le persone danno meno nei loro rapporti, possono percepire un calo del loro valore relazionale, con una conseguente svalutazione della stima di sé (Leary e Baumeister, 2000).

La responsività potrebbe inoltre portare ad un aumento della stima di sé per almeno due ragioni. In primo luogo, quando le persone sono sensibili, vengono ricambiate a loro volta con altrettanta sensibilità (es. Canevello e Crocker, 2010; Kenny e Acitelli, 2001) il che dovrebbe aumentare l'autostima (Lemay, Clark e Feeney, 2007). In secondo luogo, questi individui fanno la differenza nella la vita degli altri, con conseguente aumento della considerazione di sé (Leary e Baumeister, 2000).

Coerentemente con quanto detto sinora, Canevello e Crocker (2010) hanno svolto una ricerca su alcuni studenti del college che avevano appena iniziato a vivere insieme nella stessa stanza. I risultati emersi sottolineano che, in funzione degli obiettivi che perseguono (compassionevoli o di immagine di sé), gli studenti creano la qualità delle relazioni con i loro compagni di stanza. Quando gli studenti si sono concentrati a difendere o migliorare la loro immagine, hanno creato dinamiche di reattività negativa che alla fine hanno indotto i loro compagni a valorizzarli di meno. Invece, quando gli studenti si sono concentrati sul sostenere i compagni di stanza, hanno creato dinamiche di reattività positiva che hanno predisposto i loro compagni a dargli maggior valore.

Da un'altra ricerca di Canevello e Crocker (2011b), sempre sugli studenti universitari, è emerso che gli obiettivi compassionevoli contribuivano alla diminuzione della loro angoscia attraverso il sostegno che fornivano agli altri studenti. Invece, gli obiettivi collegati all'immagine di sé permettevano di ricevere meno sostegno dagli altri. Aumentare il dare prediceva una diminuzione dell'angoscia nel lungo periodo; invece, i vantaggi di un 
maggiore sostegno ricevuto sono stati evidenti solo nel breve periodo, suggerendo che dare sostegno ha conseguenze più sostanziali per diminuire l'angoscia.

Questa differenza tra il dare e ricevere sostegno si evince bene dagli studi che hanno messo in evidenza la relazione tra benessere psicologico e volontariato. Ad esempio, in uno studio di correlazione di Thoits e Hewitt (2001), il numero delle ore di lavoro dei volontari è stato valutato come predittivo per cambiamenti positivi nel benessere, nella felicità, nella soddisfazione di vita, nell'autostima, nella padronanza, nella depressione e nella salute fisica nell'arco di 3 anni. Inoltre, dati provenienti dalla letteratura sul soccorso e il volontariato suggeriscono che dare ha conseguenze positive per il benessere psicologico (cf. Post, 2007 per una rassegna) e per la diminuzione della mortalità (Musick, Herzog e House, 1999; Oman, Thoresen e McMahon, 1999).

Altri studi dimostrano i benefici superiori del provare compassione verso altri piuttosto che ricevere sostegno compassionevole.

Per esempio, in uno studio correlazionale condotto da Schwartz e colleghi (2003), emerse che la persona compassionevole sperimentava anche una maggiore salute mentale (meno depressione e ansia) rispetto a colui che invece era il destinatario di questi comportamenti. Inoltre, Dunn, Aknin e Norton (2008) trovarono che gli individui che avevano, in modo casuale, il compito di spendere una quota di denaro per gli altri esperivano più felicità di quelli incaricati a spenderne per se stessi.

Indipendentemente dalla quantità di sostegno ricevuto, dando supporto vi è una migliore salute mentale nella transizione tra l'adolescenza e la prima età adulta (Crocker et al., 2010) e negli anziani che si trovano ad affrontare la perdita del partner (Brown et al., 2008).

Dare supporto, anche tenendo in considerazione il supporto ricevuto, predice una pressione sanguigna più bassa, una migliore condizione di salute in generale (Brown, Consedine e Magai, 2005; Piferi e Lawler, 2006) e una mortalità più bassa (Brown et al., 2003; Brown et al., 2009).

I risultati delle ricerche, pertanto, suggeriscono che avere come obiettivo l'immagine di sé, produca nell'individuo una focalizzazione su come ottenere dagli altri un riconoscimento per le proprie qualità positive e questo, paradossalmente, ha come conseguenze una minor considerazione dagli altri, una minor autostima e una serie di conseguenze negative a livello fisico e psicologico. Al contrario, gli obiettivi compassionevoli, incentrati sull'essere di supporto agli altri, portano ad una maggior considerazione da parte loro, una maggiore autostima e numerose conseguenze positive a livello fisico e psicologico.

Un modo particolare di concettualizzare questo rapporto tra obiettivi 
interpersonali focalizzati sull'immagine di sé e quelli focalizzati sulla compassione è quello offerto da Crocker e Canevello (2008) che parlano di obiettivi eco-sistemici e obiettivi ego-sistemici.

Il desiderio e il bisogno di appartenenza al proprio sistema di relazioni, di valori e di norme comporta due distinti processi per ricercare di soddisfare questi obiettivi: aumentare la percezione di inclusione, l'accettazione, lo status (es. Leary e Baumeister, 2000); creare relazioni, sostegno e cura reciproca con gli altri, sostenendo questi rapporti nel tempo (Cohen, Gottlieb e Underwood, 2000; Uchino, 2004). Questi due tipi di obiettivi, in genere, riflettono diversi punti di vista motivazionali nel rapporto con gli altri: le prospettive egosistemiche, fondate sull'immagine di sé e le prospettive ecosistemiche, fondate sulla compassione.

Le persone che si pongono generalmente obiettivi collegati all'immagine di sé vedono il rapporto tra sé e gli altri da un punto di vista motivazionale egosistemico. Ciò significa che si concentrano su se stessi, sui propri bisogni e sui propri desideri, considerando la relazione tra sé e gli altri come un rapporto "a somma zero" (dove il guadagno di una persona corrisponde alla sconfitta di un'altra). Nella prospettiva motivazionale egosistemica i soggetti valutano e giudicano gli altri e se stessi, aspettandosi la valutazione e il giudizio altrui. Questo si manifesta con un interesse verso le impressioni degli altri, con un'attenzione eccessiva rivolta su di sé e con l'ansia sociale. È presente una forte tendenza a mettersi alla prova, a dimostrare le qualità desiderate, a cercare di convalidare il valore personale e di stabilire i propri meriti. In questo quadro, ovviamente, la priorità è per i propri bisogni rispetto a quelli degli altri.

Costruire, gonfiare, mantenere e difendere l'immagine di sé desiderata diventa un mezzo per soddisfare la propria esigenza di convincere gli altri del proprio valore. Una prospettiva egosistemica, così, cattura solo un pezzo limitato della realtà. In quest'ottica, tali persone si concentrano sugli altri solo nella misura in cui questi possono negargli o dargli qualcosa: per esempio approvazione, inclusione o convalida (Leary e Baumeister, 2000), oppure guadagni (o perdite) provenienti dall'associazione o dal confronto con gli altri (Tesser, 1988).

Situazioni interpretate come minacciose, competitive o a somma zero, attivano un punto di vista motivazionale egosistemico. In queste situazioni le persone facilmente confondono se stessi con l'immagine di sé, interpretando e rispondendo alle minacce in relazione alle immagini desiderate, come se fossero in gioco il benessere o la sopravvivenza. Questi soggetti vedono il proprio comportamento come una risposta necessaria alla situazione in cui si trovano e non pensano a come questo crei o plasmi le situazioni che esperiscono, in particolare gli aspetti spiacevoli di quelle situazioni. 
La prospettiva ecosistemica è quella assunta dalle persone compassionevoli. In biologia, un ecosistema consiste nella comunità di varie specie e nell'ambiente fisico nel quale vivono, considerati come un tutt'uno. In un ecosistema sano, varie specie soddisfano bisogni biologici gli uni degli altri per nutrimento, ossigeno, anidride carbonica, luce, ombra e così via. Questo crea un delicato equilibrio di vita reciprocamente interdipendente. Un danno a un solo elemento dell'ecosistema potrebbe influire negativamente in tutte le specie nell'ecosistema.

Nella prospettiva ecosistemica creare una fitta rete di relazioni di supporto dipende dall'esternare la propria vulnerabilità ed essere di sostegno agli altri e ai loro bisogni (cf. Reis, Clark e Holmes, 2004 per una rassegna). Le persone si sentono vicine e collegate a chi percepiscono essere comprensivo e di sostegno (LeMay, Clark e Feeney, 2007). A loro volta, i sentimenti di vicinanza favoriscono il desiderio di dare sostegno agli altri (Brown e Brown, 2006). Quindi le persone che danno supporto ai bisogni altrui, costruiscono rapporti autentici in cui alla fine ricevono sostegno. Questo accade anche quando ottenere un sostegno non è l'obiettivo ricercato intenzionalmente (Clark, Mills e Powell, 1986).

Con una prospettiva ecosistemica le persone hanno come priorità $\mathrm{i}$ bisogni degli altri, non per virtù o sacrificio, ma perché percepiscono le connessioni reciproche tra le varie persone. Con questo tipo di presupposti, gli individui si sentono liberi ma allo stesso tempo collegati agli altri in modo intersoggettivo. Questi sentimenti elevano e ispirano a migliorare, crescere ed espandere le proprie capacità (Haidt, 2003).

Un punto di vista ecosistemico, riesce a inquadrare un'area più grande di realtà rispetto a quello egosistemico. All'interno di queste prospettive, gli individui vedono il proprio comportamento come punto di partenza per la creazione di un sistema sano, o malsano, attraverso gli effetti a catena positivi o negativi dei propri comportamenti. Vedono e si assumono la propria responsabilità nel creare le relazioni e le condizioni che desiderano.

Invece di proteggere se stessi e di competere con gli altri per ottenere ciò che vogliono, ritengono di poter collaborare con altri per creare risultati positivi e, prendendo in considerazione i bisogni altrui, creano la migliore possibilità per soddisfare le proprie esigenze. Tali individui ritengono sia importante che le persone si prendano cura le une delle altre.

\subsubsection{Compassione per sé nella relazione con l'altro}

Le persone con auto-compassione sono più propense a risolvere i con- 
flitti relazionali in modo che le esigenze di sé e degli altri siano bilanciate. I partecipanti a un esperimento di Yarnell e Neff (2013) - 506 studenti universitari americani di cui 267 femmine e 239 maschi di età compresa tra i 17 e i 24 anni - che avevano più alta auto-compassione avevano significativamente più probabilità di arrivare a un compromesso relazionale anziché subordinare le loro esigenze nei conflitti con le madri, i padri, i migliori amici e i loro partner. Questo suggerisce che questi individui tendono a valorizzare le esigenze e i desideri di se stessi e dei loro partner, permettendosi di risolvere i conflitti in modo sano e produttivo.

I partecipanti all'esperimento di Yarnell e Neff che avevano un'alta auto-compassione presentavano maggiori probabilità di riportare una sensazione di autenticità quando risolvevano $\mathrm{i}$ conflitti relazionali (in tutti $\mathrm{i}$ contesti) rispetto a quelli che non avevano compassione di sé. La capacità delle persone con auto-compassione di accettare se stessi consentiva loro di agire in conformità con i loro pensieri e valori interiori (Neff, 2003a; Neff, Hseih e Dejitthirat, 2005) e, quindi, di affermarsi in modo autentico nelle relazioni. Gli studenti auto-compassionevoli segnalavano inoltre di avvertire meno turbamento emotivo quando cercavano di decidere come risolvere i conflitti relazionali. Quando sorgono spinosi problemi di relazione, l'auto-compassione può aiutare le persone a lenire e calmare l'intensità delle emozioni percepite, in modo che si possa rispondere ai conflitti più serenamente (Kelly, Zuroff e Shapira, 2009). Allo stesso modo, i risultati hanno indicato che una maggiore auto-compassione era collegata a un maggior senso di benessere all'interno delle relazioni. L'auto-compassione è stata costantemente associata anche con una maggiore capacità di ricupero emotivo (Neff, 2009) e questo potrebbe aiutare anche nella gestione delle emozioni derivanti dai conflitti.

\subsection{Autostima e auto-compassione: gli effetti sul benessere soggettivo}

In generale è emerso, che le persone con maggiore auto-compassione sono meno propense a sperimentare esiti psicologici negativi (Leary et al., 2007; Neff, 2003a). Inoltre, Neff, Kirkpatrick e Rude (2007) hanno trovato che l'auto-compassione era un predittore significativo di felicità, speranza e interessi positivi.

Un corpo convergente della letteratura suggerisce che le varie forme di comportamento compassionevole hanno benefici immediati per la salute psicologica ma anche a lungo termine, tra cui ad esempio stati d'animo positivi (Millar, Millar e Tesser, 1988) riduzione dei sintomi depressivi (Krause, Herzog e Baker, 1992; Taylor e Turner, 2001) e una maggiore autostima 
(Krause e Shaw 2000; Yogev e Ronen, 1982). I comportamenti basati sulla compassione possono anche provvedere a fornire meccanismi di coping in occasioni di momenti stressanti della vita: è stato riscontrato, ad esempio, che individui con un maggior numero di esperienze di compassione sperimentavano maggiore sostegno sociale (Crocker e Canevello, 2008).

Secondo Crocker e Park (2004), le contingenze che attivano l'autostima e il suo perseguimento sono difficili da cambiare, perché fondate sulle prime esperienze emotive infantili che, quindi, potrebbero non essere accessibili alla coscienza. Al contrario, ci si può porre intenzionalmente altri obiettivi che siano buoni per sé e per gli altri. In questi obiettivi intenzionali rientra l'auto-compassione.

Gli obiettivi collegati alla compassione non possono sedare ansie e paure, come fa l'autostima, ma forniscono una motivazione per proseguire nel loro raggiungimento per il valore intrinseco che hanno. Tuttavia, lasciare gli obiettivi collegati all'autostima richiede di far fronte alle proprie paure e ansie circa la morte, l'abbandono, il rifiuto o l'incompetenza. Inoltre, perseguire i propri obiettivi nonostante questi timori, richiede forte motivazione che deriva dall'avere obiettivi nei quali si crede profondamente.

Gli obiettivi legati all'auto-compassione danno più autonomia perché il comportamento non è guidato da preoccupazioni inerenti l'autostima (Deci e Ryan, 1995). Per questo, facilitano l'apertura all'apprendimento e, includendo anche altre persone, sono più relazionali.

È interessante notare che la conseguenza di passare da obiettivi di autoconvalida ad obiettivi che includono gli altri, paradossalmente, sembra produrre un'autostima più stabile, non contingente e non difensiva (Deci e Ryan, 1995; Dweck, 2000; Kernis, 2003).

L'auto-compassione comporta molti dei benefici psicologici che sono stati associati alla ricerca di alta autostima, presentando meno insidie. Infatti, non basandosi sulle valutazioni delle prestazioni di sé e degli altri, aiuta a innescare comportamenti protettivi contro gli effetti debilitanti dell'eccessivo giudizio su di sé (Horney, 1950), come ad esempio la depressione e l'ansia (Blatt et al., 1982). Inoltre, contrasta le tendenze verso il narcisismo e l'egocentrismo che possono eventualmente derivare dal tentativo di mantenere alta l'autostima (Finn, 1990; McMillan, Singh e Simonetta, 1994), rafforzando i sentimenti di connessione con gli altri, piuttosto che ponendosi in opposizione ad essi.

Entrando nel vivo di alcune ricerche, in uno studio svolto in Australia su 2448 soggetti adolescenti (49,6\% femmine e 50,4\% maschi), è emerso che tra coloro che avevano un alto livello di auto-compassione, la bassa autostima aveva avuto poca influenza sulla salute mentale. Al contrario, tra quelli a basso contenuto di auto-compassione, la bassa autostima 
faceva prevedere cali significativi nei punteggi di salute mentale (Marshall et al., 2015).

Neff e Vonk (2009) hanno svolto una ricerca sul rapporto tra compassione e autostima su un campione composto da 2.187 soggetti (26\% uomini e 74\% donne). Lo studio ha evidenziato che l'auto-compassione permette di prevedere una maggiore stabilità emotiva rispetto all'autostima ed è meno suscettibile a variare in funzione di particolari risultati contingenti. Inoltre, è risultata correlata negativamente con il confronto sociale, l'autocoscienza pubblica (preoccuparsi della propria immagine agli occhi degli altri), la ruminazione, la rabbia e il bisogno di eliminare dalla consapevolezza alcune informazioni incongruenti con la propria visione delle cose.

Come già osservato in precedenza, uno dei maggiori problemi potenziali di un'alta autostima è che essa può manifestarsi come narcisismo. In linea con precedenti ricerche (Neff, 2003a), anche dalla ricerca di Neff e Vonk (2009) è emerso che l'autostima ha una sostanziale associazione positiva con il narcisismo, mentre l'associazione tra auto-compassione e narcisismo è vicina allo zero. Presumibilmente, le persone con alta auto-compassione non hanno bisogno di dilatare il proprio concetto di sé dato che possono accettare i loro punti deboli e di forza. Va considerato che le misure standard del tratto di autostima (es. Berger, 1952; Rosenberg, 1965) correlano positivamente con punteggi sulla Narcissistic Personality Inventory (Raskin e Hall, 1979), cosa che con l'auto-compassione invece non accade (Neff, 2003b).

In un altro studio di Neff e Vonk (2009), si è visto che l'auto-compassione, rispetto all'autostima, in un arco temporale di otto mesi garantiva una maggiore stabilità del proprio valore personale. Anche in questo caso $\mathrm{i}$ risultati hanno indicato che l'auto-compassione, rispetto all'autostima globale, ha una forte relazione inversa con il confronto sociale, la coscienza pubblica di sé e la ruminazione. È possibile che avere compassione per se stessi quando nascono sentimenti di inadeguatezza sia legato a un senso di calma e di sicurezza (Gilbert e Irons, 2005). Questo implica che le risorse attentive siano meno orientate a preoccuparsi di ciò che gli altri pensano, o nel fissarsi ossessivamente su se stessi in quanto buoni o cattivi. Ciò sarebbe coerente con ricerche precedenti in cui si è vista un'associazione negativa tra la compassione, la ruminazione e l'ansia (es. Neff, 2003a; Neff, Kirkpatrick e Rude, 2007).

Neff (2006) ha riscontrato che l'auto-compassione risulta essere più predittiva di comportamenti relazionali positivi rispetto all'autostima per ciò che concerne la cura, l'intimità, il sostegno e atteggiamenti relazionali non aggressivi e romantici (come riportato dai partner). Ha anche dimostrato che l'autostima complessiva e l'auto-compassione sono moderatamente correlate (Neff, 2003a), ma è l'auto-compassione a predire in modo unico i 
livelli di depressione e di ansia. In una ricerca condotta da Neff, Kirkpatrick e Rude (2007) si richiedeva ai soggetti di svolgere un compito che metteva in discussione un aspetto di debolezza soggettiva. Si è rilevato che l'auto-compassione era associata a una riduzione dell'ansia. Al contrario, l'autostima non è risultata altrettanto protettiva.

Anche se l'autostima tende a farsi sentire quando le cose vanno bene e l'auto-compassione è più rilevante quando le cose vanno male, questi risultati suggeriscono che l'auto-compassione è legata anche a stati emotivi positivi. Le ragioni per cui l'auto-compassione e l'autostima sono associate alle emozioni positive, però, sono diverse.

Gli individui con autostima alta possono sentirsi felici, ottimisti e fiduciosi perché si valutano positivamente. Inoltre, i risultati self-report dell'autostima possono essere influenzati dal benessere generale perché le persone spesso usano l'euristica «mi sento bene e quindi sto bene con me stesso» (Brandt e Vonk, 2006). L'alta stima di sé è stata associata inoltre con illusioni egoistiche e insufficiente auto-regolazione (Baumeister, Heatherton e Tice, 1993).

Individui con alti livelli di auto-compassione, invece, con più probabilità hanno sentimenti positivi perché si accettano per quello che sono. In questo modo temono di meno un possibile attrito con il fallimento, il rifiuto e i propri limiti, che costituiscono parte inevitabile della vita reale. Inoltre, avere compassione per se stessi comporta che lo stato d'animo negativo associato con la sofferenza è parzialmente trasformato in uno stato affettivo più positivo. Uno stato d'animo positivo, a sua volta, è stato associato con una maggiore attenzione e con una più accurata elaborazione di informazioni rilevanti per il sé (Aspinwall, 1998). Questo perché le persone non devono nascondere a se stesse i propri difetti e carenze al fine di evitare di giudicarsi (es. Reis et al., 2000).

A differenza di un'alta stima di sé, che può portare all'adozione di obiettivi inadeguati che vanno oltre le proprie capacità, l'auto-compassione dovrebbe essere collegata a una maggiore conoscenza e chiarezza circa $i$ propri limiti. Non sorprende, quindi, che le persone auto-compassionevoli tendano ad avere alta autostima (Neff, 2003b), presumibilmente perché reagire gentilmente anziché in modo critico verso se stessi promuove sentimenti positivi nei propri riguardi. Tuttavia, le sensazioni positive che caratterizzano le persone con auto-compassione non sembrano coinvolgere arroganza, narcisismo oppure desideri eccessivi o illusori di auto-miglioramento che invece caratterizzano molte persone che possiedono, o desiderano avere, un'alta autostima.

Cinque studi svolti da Leary e colleghi (2007) hanno esaminato i processi cognitivi ed emotivi con i quali i soggetti con auto-compassione af- 
frontano gli eventi della vita spiacevoli, in comparazione con i risultati dovuti all'autostima. Nei vari studi, condotti su studenti di psicologia con età compresa tra i 17 e i 23 anni, i partecipanti riferivano circa il loro modo di vivere e affrontare eventi negativi della loro vita quotidiana, rispondendo a scenari ipotetici, reagendo a feedback interpersonali, valutando le loro e altrui performances videoregistrate in una situazione imbarazzante e riflettendo su esperienze personali negative: è emerso che i partecipanti con elevata auto-compassione hanno indicato con più forza di aver cercato di essere gentili e meno duri con se stessi a seguito di eventi negativi.

È stato inoltre rilevato, che l'auto-compassione era inversamente correlata al pensiero che i propri problemi siano più grandi rispetto agli altri e che la propria vita è più problematica di quella di altre persone. Invece, è risultata positivamente correlata alla convinzione che l'evento negativo non sia peggio di ciò che accade ad altri.

Rispetto all'attribuzione interna degli errori, l'auto-compassione è risultata associata con il guardare la situazione in prospettiva, non avallando l'idea di essere un "perdente", producendo emozioni negative più basse e riducendo la sensazione che qualcuno avrebbe gestito la situazione meglio. L'auto-compassione era positivamente correlata col tentativo di capire le proprie emozioni in quegli eventi nei quali i partecipanti indicavano di aver commesso degli errori e inversamente correlata alle emozioni auto-coscienti dopo gli eventi nei quali ritenevano di non avere responsabilità.

Analizzando la correlazione tra reazione soggettiva ed eventi negativi, è emerso anche che l'auto-compassione prediceva in modo unico le reazioni agli scenari negativi con meno catastrofizzazione, meno personalizzazione e maggiore equanimità, anche rispetto ad eventi negativi immaginati. Questo risultato è stato particolarmente interessante per i partecipanti con basso livello di autostima.

Gli Autori hanno anche scoperto che l'auto-compassione ha permesso alle persone di riconoscere il loro ruolo negli eventi negativi, mentre mantenevano una prospettiva più ampia sui concetti di sé negativi. Importa sottolineare come le persone con auto-compassione sembravano più in grado di prendere una posizione che accettava ed era aperta agli aspetti indesiderati di sé, senza coinvolgersi necessariamente in pensieri negativi e comportamenti difensivi.

Considerando il confronto tra auto-compassione e autostima, durante l'analisi dei dati entrambi i costrutti sono stati inseriti simultaneamente nell'analisi della regressione. I risultati hanno dimostrato che l'auto-compassione aveva una varianza unica relativamente ai risultati, mentre l'autoautostima no. Più importante, se si fosse misurato unicamente il peso del- 
l'autostima, probabilmente si sarebbe concluso che tutti gli effetti positivi evidenziati erano stati a causati da essa, ignari che l'auto-compassione poteva essere il fattore importante a influenzare i risultati. Questi dati sollevano la possibilità che molti effetti attribuiti all'autostima, in realtà, possano essere piuttosto una funzione dell'auto-compassione.

Analizzando il rapporto tra autostima e auto-compassione con gli stimoli neutri, è emerso che i partecipanti a basso contenuto di auto-compassione hanno reagito in modo diverso agli stimoli neutri o ambivalenti rispetto ai partecipanti ad alto contenuto di auto-compassione. Invece, le persone ad alto contenuto di auto-compassione hanno reagito in modo simile ai due tipi di stimoli. I partecipanti con più alta autostima erano meno propensi ad attribuire le risposte neutre a se stessi rispetto a quelli con più bassa autostima. L'auto-compassione, invece, è stata associata con la propensione ad accettare che il feedback negativo poteva essere causato dalla propria personalità. Generalizzando, piuttosto che negare di poter aver causato i risultati negativi della vita, le persone auto-compassionevoli sono aperte ad assumersi un certo livello di responsabilità personale. Tuttavia, anche quando si assumono tali responsabilità, tenendo conto delle ricerche descritte in precedenza, non sembrano rimuginare sulle possibili implicazioni negative.

Analizzando le relazioni tra autostima e autovalutazioni sulle performance è emerso che l'alta autostima è associata con valutazioni esagerate sulle proprie performance e caratteristiche rispetto a osservatori esterni, mentre la bassa autostima a valutazioni di sé più basse rispetto a quanto fanno osservatori esterni (cf. anche Robins e Beer, 2001).

Analizzando gli stili di attribuzione in merito agli eventi negativi è emerso che i soggetti con alta auto-compassione, hanno uno stile di attribuzione più interno. Nello specifico, riescono a considerare come causa il loro modo di essere. Inoltre, queste persone esperiscono meno emozioni negative di fronte ai propri errori: questo perché si ha la capacità di riconoscere di poter commettere errori ma senza sentirsi in difetto per questo.

Partendo dal presupposto che le persone spesso mantengono sentimenti positivi verso di sé attraverso illusioni, (Blaine e Crocker, 1993; Leary, 2004; Murray, Holmes e Griffin, 1996; Taylor e Brown, 1988), è importante considerare che tali illusioni sono spesso mantenute debolmente, circondate da un certo grado di auto-inganno e a volte sostenute nonostante i giudizi critici di altre persone. Suddette illusioni non solo compromettono l'abilità delle persone a vedere se stesse e gli altri in modo accurato (Leary, 2004, 2007), ma a volte sono anche impossibili da sostenere di fronte alle prove incontrovertibili delle proprie mancanze. Queste strategie appena descritte, sono caratteristiche di soggetti con elevata autostima. Al contrario, un orientamento auto-compassionevole non richiede illusioni o un atteggiamento difensivo ma 
piuttosto implica una chiara percezione delle caratteristiche personali, buone e cattive che siano. In questo modo l'auto-compassione permette di riconoscere i propri difetti, pur rimanendo gentili e comprensivi verso se stessi, favorendo percezioni precise e, quindi, un comportamento più efficace.

Da una ricerca di Johnson e O'Brien (2013) svolta su un campione di 335 studenti universitari (di età inferiore ai 40 anni) è emerso che, in linea con le affermazioni teoriche (Neff, 2003a, 2011) e l'evidenza empirica (Leary et al., 2007; Neff, 2003b) l'auto-compassione è risultata significativamente e sostanzialmente associata a sintomatologia depressiva più bassa. Inoltre, è stato il primo studio randomizzato mirato a verificare se gli individui inclini alla vergogna possano trarre vantaggio dall'utilizzo dell'autocompassione per elaborare esperienze che provocano vergogna. I risultati mostrano che la pratica attiva di auto-compassione nei confronti di ricordi che suscitano vergogna produce risultati positivi sia immediatamente che nelle due settimane successive.

Livelli più elevati di auto-compassione sono stati associati con una maggiore soddisfazione di vita, l'intelligenza emotiva, l'iniziativa personale, la competenza percepita, la felicità, l'attaccamento sicuro, la connessione sociale e anche minor autocritica, depressione, ansia, ruminazione, soppressione pensiero, perfezionismo, obiettivi di performance e disturbi del comportamento alimentare (Adams e Leary, 2007; Neff, 2003a, Neff, Hseih e Dejitthirat, 2005; Neff, Kirkpatrick e Rude, 2007; Neff, Pisitsungkagarn e Hseih, 2008; Neff e McGehee, 2010).

Neff, Rude e Kirkpatrick (2007) hanno trovato che l'auto-compassione è stata associata a un aumento dei livelli di consapevolezza riflessiva e affettiva, iniziativa personale, curiosità ed esplorazione, felicità e ottimismo. Essi hanno anche scoperto che l'auto-compassione era significativamente associata con l'estroversione, la gradevolezza, la coscienziosità e negativamente con il nevroticismo. L'auto-compassione ancora presumeva una varianza unica nel funzionamento positivo dopo aver controllato per le variabili di personalità.

È importante sottolineare che, l'auto-compassione non è associata a un pregiudizievole aumento della stima di sé (Neff, 2003a; Neff e Vonk, 2009), ma è associato con l'assunzione di una maggiore responsabilità per gli errori del passato (Leary et al., 2007). Ciò suggerisce che l'auto-compassione non è solo un modo di "distorcere" azioni o eventi passati o presenti al fine di vedere se stessi in modo positivo. Ha dimostrato inoltre di avere validità discriminante per quanto riguarda le altre misure di adattamento psicologico, come l'autostima (Neff, 2003a), l'attaccamento sicuro (Neff e McGehee, 2010), le influenze negative (Neff, Kirkpatrick e Rude, 2007) e i tratti di personalità del "Big Five" - piacevolezza, 
estroversione, coscienziosità, apertura all'esperienza e nevroticismo (Neff, Maleducato e Kirkpatrick, 2007).

Neff, Hsieh e Dejitterat (2005) hanno esaminato la relazione tra la compassione di sé e le reazioni al fallimento accademico. Tra gli studenti che avevano ricevuto un'insoddisfacente valutazione sul "medio termine", l'autocompassione correlava positivamente con le strategie di coping di accettazione e la reinterpretazione positiva/crescita e correlava negativamente col focus sulle emozioni negative e con l'evitamento emotivo. Inoltre, l'autocompassione era positivamente associata con un orientamento alla padronanza (essendo motivata dalla curiosità e dal desiderio di sviluppare le proprie competenze) e associata negativamente con l'orientamento ai risultati (la motivazione a difendere o migliorare la propria autostima).

$\mathrm{Da}$ quanto detto finora emerge che le persone con auto-compassione, dovrebbero evidenziare una maggiore salute psicologica rispetto a quelle con bassi livelli di auto-compassione, perché il dolore inevitabile e il senso di fallimento, vissuto da tutti gli individui, non viene amplificato e perpetuato attraverso una dura auto-condanna, con sentimenti di isolamento e con sovra-identificazione con i pensieri e le emozioni. L'auto-compassione dovrebbe essere correlata alla capacità di guardare e gestire le proprie emozioni, piuttosto che a evitarle o negarle. Inoltre, se gli individui con autocompassione hanno una maggiore autoconoscenza, dovrebbero essere anche in grado di individuare i modi in cui le loro azioni contribuiscono a mantenere o aggravare una situazione stressante, consentendo loro di intraprendere anche "passi più focalizzati" per alleviare lo stress.

\subsection{Immagine di sé e processi fisiologici}

Le prove suggeriscono che gli obiettivi riferiti al miglioramento o al mantenimento dell'immagine di sé e gli obiettivi compassionevoli implicano processi fisiologici distinti. In particolare, gli obiettivi di auto-immagine possono attivare il sistema di autoconservazione, di "lotta o fuga" incentrato sulla sopravvivenza individuale, regolato dagli ormoni dello stress come il cortisolo (Henry e Wang, 1998). La risposta di lotta o fuga coinvolge parti del cervello evolutivamente più antiche e si trova in molte specie, tra cui i rettili. L'attivazione a breve termine della risposta di lotta o fuga mobilita risorse individuali e facilita risposte adattative alle minacce immediate di sopravvivenza. Poiché le risposte alle minacce di vita o di morte devono essere veloci, questo sistema non favorisce risposte riflessive, analitiche o empatiche, che richiedono un tempo che potrebbe costare la vita. L'attivazione di questo sistema comporta un beneficio per la so- 
pravvivenza individuale nel breve termine; ma l'attivazione prolungata risulta costosa per la salute fisica e mentale (Sapolsky, 1998).

Anche le minacce psicologiche, reali o percepite, possono attivare il sistema di auto-conservazione (cf. Dickerson e Kemeny, 2004 per una rassegna). In particolare, le minacce all'immagine di sé hanno favorito la produzione di cortisolo in alcuni esperimenti di laboratorio. La ricerca mostra anche che le minacce di vario genere all'immagine di sé diminuiscono la capacità di pensare razionalmente (Baumeister, Twenge e Nuss, 2002; Steele, Spencer e Aronson, 2002) e di auto-regolazione (Twenge, Catanese e Baumeister, 2002), diminuiscono il comportamento prosociale (Twenge, Baumeister et al., 2007) e istigano all'aggressività (Bushman e Baumeister, 1998; Twenge et al., 2001).

Sulla base di questi presupposti teorici, è ipotizzabile che le minacce all'auto-immagine attivino la risposta di lotta o fuga perché confondono le minacce all'immagine di sé con minacce alla sopravvivenza. Quando le persone sperimentano una minaccia all'immagine di sé e reagiscono con una risposta di fuga o di lotta, usano parti del cervello che si sono evolute per affrontare le minacce di vita o di morte. Invece, risposte efficaci alle minacce all'immagine di sé richiederebbero di capire l'intenzione dell'altro. Questo comporta anche la necessità di considerare l'effetto della propria risposta sull'altra persona e le conseguenze di tutto questo per il raggiungimento degli obiettivi a lungo termine, astenendosi ovviamente dalle risposte che minano questi obiettivi, per quanto buone possano apparire al momento e tutto ciò richiede capacità evolutivamente più recenti.

Inoltre, analizzando le reazioni auto-compassionevoli rispetto all'immagine di sé, è ipotizzabile che gli obiettivi compassionevoli attivino il sistema "prenditi cura e sii amichevole", aumentando le possibilità di sopravvivenza degli altri favorendo comportamenti protettivi e assistenziali. Questo sistema è regolato da ormoni riproduttivi quali ossitocina, vasopressina e progesterone (Henry e Wang, 1998; Taylor et al., 2000): l'ossitocina, in particolare, accresce la percezione di sentimenti di fiducia, generosità e amore. L'attivazione di questo sistema comporta benefici per la sopravvivenza a lungo termine degli individui, della loro progenie e dei loro gruppi sociali favorendo relazioni di sostegno e l'auto-regolazione.

\section{Conclusioni}

Dalle molteplici ricerche che abbiamo analizzato e descritto, risulta evidente, a nostro avviso, quanto avere la stima di sé come obiettivo centrale o prioritario nella vita sia altamente rischioso se non addirittura contro- 
producente; senza fornire, tra l'altro, la garanzia di risultati duraturi. Invece, questi ultimi sono continuamente messi a rischio dall'autocritica, dal dubbio e dagli eventi della vita, dando origine a una ricerca costante di conferme o di successi per raggiungere o mantenere il livello di autostima desiderato, con crescenti conseguenze negative a livello di salute fisica, benessere emotivo e qualità delle relazioni interpersonali.

Abbiamo invece visto come avere la compassione, per sé o per l'altro, come obiettivo primario o prioritario, permette di raggiungere quegli obiettivi promessi dalla ricerca dell'autostima (ad esempio una buona valutazione di sé e un senso di inclusione sociale), in modo più stabile, senza conseguenze negative direttamente riconducibili a essa (nel senso che, ad esempio, non si può dare alla compassione verso l'altro la responsabilità dell'uso che fa l'altro della compassione ricevuta, tradendo la nostra fiducia) e, anzi, con una serie di conseguenze positive a livello personale e interpersonale.

L'auto-compassione, inoltre, e non l'autostima, è collegata positivamente con la capacità di comprendere se stessi, gli altri e gli eventi, traendo costantemente nuovi spunti per migliorare sé, gli altri, le relazioni e le situazioni. Cercare di migliorare o sostenere il concetto di sé sembra essere un obiettivo condiviso da tantissime persone e probabilmente è il residuo, in età adulta, di bisogni infantili mai completamente soddisfatti da piccoli, come il bisogno di sentirsi riconosciuti, amati e importanti da chi si è preso cura di noi, permettendoci di passare così: dal sentirci riconosciuti e protetti per quello che facciamo (ad esempio comportarci secondo le aspettative dei genitori, oppure le gratificazioni che riceviamo per cose fatte bene o belle), al sentirci tali per quello che siamo (ad esempio figli e poi esseri umani degni di valore in sé); e, infine, al riconoscere noi stessi di esserlo, senza più necessità di conferme; per poi passare, in unltima analisi, al dare a noi stessi e agli altri quel riconoscimento che abbiamo avuto modo di sperimentare e che ora risiede dentro di noi in modo stabile.

Così, come arriva un'età nella quale apprendiamo la cosiddetta "costanza dell'oggetto", ne arriva un'altra nella quale la stessa abilità la applichiamo al valore personale, che percepiamo con costanza anche in assenza di conferme costanti.

Di conseguenza, quando la nostra mente ci propone come obiettivo quello di convalidare, rinforzare o aumentare la percezione del nostro valore personale, la risposta migliore che possiamo dare a questa richiesta è quella di dedicarci con cura a noi stessi e agli altri. 


\section{Sommario}

In questo contributo analizzeremo due costrutti psicologici: l'autostima e l'auto-compassione, secondo quanto scritto in letteratura. In particolare, confronteremo gli effetti dell'alta autostima e della sua continua ricerca con quelli l'autocompassione, evidenziando i nuovi contributi teorici che sottolineano gli effetti negativi del primo costrutto confrontarli con quelli produttivi del secondo.

Esamineremo l'alta autostima e l'auto-compassione in rapporto agli aspetti relazionali, psicologici, emotivi e di performance dell'individuo e della comunità. Nelle conclusioni apriremo uno spazio di riflessione che mira a rivalutare l'obiettivo frequente di ricercare costantemente un'alta autostima e di integrare quest'ultima con una cura di se stessi e degli altri.

Parole chiave: autostima, auto-compassione, relazioni interpersonali, emozioni.

\section{Abstract. Self-esteem and self-compassion. Two different ways of relating to themselves}

In this paper we will analyze two psychological constructs: self-esteem and self-compassion, as written in literature. In particular, we will compare the effects of the high self esteem and his continuous research with the self-compassion, highlighting new theoretical contributions that emphasize the negative effects of the first construct comparing them with productive ones of the second.

We will examine the high self-esteem and self-compassion in relation to relational, psychological, emotional and individual performance and community. In the conclusions we will open a space for reflection that aims to re-evaluate the common goal of seeking consistently high self esteem considering the necessity to achieve this goal thanks to the care of oneself and of others.

Key words: self-esteem, self-compassion, relationship, emotions.

\section{Riferimenti bibliografici}

Aberson C.L., Healy M. e Romero V. (2000), "Ingroup bias and self-esteem: A metaanalysis". Personality and Social Psychology Review, 4: 157-173. DOI: 10.1207/S15327957PSPR0402_04

Adams C.E. e Leary M.R. (2007), "Promoting self-compassionate attitudes towards eating among restrictive and guilty eaters", Journal of Social and Clinical Psychology, 26, 10: 1120-1144.

Aknin L.B., Dunn E.W. e Norton M.I. (2009), From wealth to well-being: Spending money on others promotes happiness. Paper presented at the Interdisciplinary Nineteenth Century Studies Conference, Saratoga Springs, NY.

Ali S.M., Merlo J., Rosvall M., Lithman T. e Lindstrom M. (2006), "Social capital, the 
miniaturisation of community, tradiationalism and first time acute myocardial infarction: A prospective cohort study in southern Sweden", Social Science and Medicine, 63: 2204-2217. DOI: 10.1016/j.socscimed.2006.04.007

Alicke M. (1985), "Global self-evaluation as determined by the desirability and controllability of trait adjectives", Journal of Personality and Social Psychology, 49: 1621-1630. DOI: 10.1037/0022-3514.49.6.1621

Allport G.W. (1954), The nature of prejudice, Cambridge, MA: Addison-Wesley.

Allport G.W. (1955), Becoming, New Haven, CT: Yale University Press.

Aspinwall L.G. e Taylor S.E. (1993), "Effects of social comparison direction, threat, and self-esteem on affect, self-evaluation, and expected success", Journal of Personality and Social Psychology, 64: 708-722. DOI: 10.1037/0022-3514.64.5.708

Aspinwall L.G. (1998), "Rethinking the role of positive affect in self-regulation", Motivation and Emotion, 22: 1-32. DOI: 10.1023/A:1023080224401

Baker L.R. e McNulty J.K. (2011), "Self-compassion and relationship maintenance: The moderating roles of conscientiousness and gender", Journal of Personality and Social Psychology, 100: 853-873. DOI: 10.1037/a0021884

Batson C.D. (1987), Prosocial motivation: Is it ever truly altruistic? Advances in Experimental Social Psychology, 20: 65-122. DOI: 10.1016/S0065-2601(08) 60412-8

Batson C.D. (1998), Altruism and prosocial behavior, in Gilbert D.T., Fiske S.T., Lindzey G. (eds.), Handbook of social psychology, 2 (4th ed.): 282-316. New York: McGraw-Hill.

Baumeister R.F, Twenge J.M. e Nuss C.K. (2002), "Effects of social exclusion on cognitive processes: Anticipated aloneness reduces intelligent thought", Journal of Personality and Social Psychology, 83: 817-827. DOI: 10.1037/00223514.83.4.817

Baumeister R.F. e Cairns K.J. (1992), "Repression and self-presentation: When audiences interfere with selfdeceptive strategies", Journal of Personality and Social Psychology, 62: 851-862. DOI: 10.1037/0022-3514.62.5.851

Baumeister R.F. e Jones E.E. (1978), "When self-presentation is constrained by the target's knowledge: Consistency and compensation", Journal of Personality and Social Psychology, 36: 608-618. DOI: 10.1037/0022-3514.36.6.608

Baumeister R.F. e Leary M.R. (1995), "The need to belong: Desire for interpersonal attachments as a fundamental human motivation", Psychological Bulletin, 117: 497-529. DOI: 10.1037/0033-2909.117.3.497

Baumeister R.F. (1998), The self, in Gilbert D.T., Fiske S.T. e Lindzey G. (eds.), Handbook of social psychology (4th ed.), 2: 680-740, New York: McGraw-Hill.

Baumeister R.F., Bushman B.J. e Campbell W.K. (2000), "Selfesteem, narcissism, and aggression: Does violence result from low selfesteem or from threatened egotism?", Current Directions in Psychological Science, 9: 26-29. DOI: 10.1111/14678721.00053

Baumeister R.F., Campbell J.D., Krueger J.I. e Vohs, K.D. (2003), “Does high selfesteem cause better performance, interpersonal success, happiness, or healthier lifestyles? “, Psychological Science in the Public Interest, 4: 1-44. DOI: 10.1111/15291006.01431

Baumeister R.F., Heatherton T.F. e Tice D.M. (1993), "When ego threats lead to selfregulation failure: Negative consequences of high self-esteem", Journal of Personality and Social Psychology, 64: 141-156. DOI: 10.1037/0022-3514.64.1.141

Baumeister R.F., Smart L. e Boden J.M. (1996), "Relation of threatened egotism to vio- 
lence and aggression: The dark side of high self-esteem", Psychological Review, 103: 5-33. DOI: 10.1037/0033-295X.103.1.5

Beach S.R. e Tesser A. (1995), Self-esteem and the extended self-evaluation maintenance model: The self in social context, in Kernis M.H. (ed). Efficacy, agency, and self-esteem, 145-170, New York: Plenum Press.

Beauregard K.S. e Dunning D. (1998), "Turning up the contrast: Selfenhancement motives prompt egocentric contrast effects in social judgments", Journal of Personality and Social Psychology, 74: 606-621. DOI: 10.1037/0022-3514.74.3.606

Bennett-Goleman T. (2001), Emotional alchemy: How the mind can heal the heart, New York: Three Rivers Press.

Benson P.L., Galbraith J. e Espeland P. (1998), What kids need to succeed: Proven, practical ways to raise good kids, Golden Valley, MN: Free Spirit Press.

Berger E.M. (1952), "The relation between expressed acceptance of self and expressed acceptance of others", Journal of Abnormal Psychology, 47: 561-571.

Berkman L.F., Glass T., Brissette I. e Seeman T.E. (2000), "From social integration to health: Durkheim in the new millennium", Social Science and Medicine, 51: 843857. DOI: 10.1016/S0277-9536(00)00065-4

Blaine B. e Crocker J. (1993), Self-esteem and self-serving biases in reactions to positive and negative events: An integrative review, in Baumeister R.F. (ed.), Selfesteem: The puzzle of low self-regard, 55-85, New York: Plenum Press.

Blatt S.J. (1995), "The destructiveness of perfectionism: Implications for the treatment of depression", American Psychologist, 50: 1003-1020. DOI: 10.1037/0003066X.50.12.1003

Blatt S.J., Quinlan D.M., Chevron E.S., McDonald C. e Zuroff D. (1982), "Dependency and self-criticism: Psychological dimensions of depression", Journal of Consulting and Clinical Psychology, 50: 113-124. DOI: 10.1037/0022-006X.50.1.113

Bowlby J. (1969), Attachment and loss: 1. Attachment, New York: Basic Books.

Bowlby J. (1973), Attachment and loss: 2. Separation: Anxiety and anger, New York: Basic Books.

Bowlby J. (1980), Attachment and loss: 3. Loss, separation, and depression, New York: Basic Books.

Bradley G. W. (1978), "Self-serving biases in the attribution process: A reexamination of the fact or fiction question", Journal of Personality and Social Psychology, 36: 56-71. DOI: 10.1037/0022-3514.36.1.56

Brandt A.C. e Vonk R. (2006), "Who do you think you are? On the link between selfknowledge and self-esteem", in Kernis M. (ed), Self-esteem: Issues and answers, 224-229, New York: Psychology Press.

Brown B. (1999), Soul without shame: A guide to liberating yourself from the judge within, Boston: Shambala.

Brown J.D. (1986), "Evaluations of self and others: Self-enhancement biases in social judgments", Social Cognition, 4: 353-376.

Brown S.L. e Brown R.M. (2006), "Selective investment theory: Recasting the functional significance of close relationships", Psychological Inquiry, 17: 1-29. DOI: 10.1207/s15327965pli1701_01

Brown S.L., Brown R.M., House J.S. e Smith D.M. (2008), “Coping with spousal loss: Potential buffering effects of self-reported helping behavior", Personality and Social Psychology Bulletin, 34: 849-861. DOI: 10.1177/0146167208314972

Brown S.L., Nesse R.M., Vinokur A.D. e Smith D.M. (2003), "Providing social support 
may be more beneficial than receiving it: Results from a prospective study of mortality", Psychological Science, 14: 320-327. DOI: 10.1111/1467-9280.14461

Brown S.L., Smith D.M., Schulz R., Kabeto M.U., Poulin M.A. e Kim e Langa K.M. (2009), "Caregiving behavior is associated with decreased mortality risk", Psychological Science, 20: 488-494. DOI: 10.1111/j.1467-9280.2009.02323.x

Brown W.M., Consedine N.S. e Magai C. (2005), "Altruism relates to health in an ethnically diverse sample of older adults", Journal of Gerontology: Series B: Psychological Sicences and Social Sciences, 60b: 143-152. DOI: 10.1093/geronb/60.3.P143

Bushman B.J. e Baumeister R.F. (1998), "Threatened egotism, narcissism, self-esteem, and direct and displaced aggression: Does self-love or self-hate lead to violence?", Journal of Personality and Social Psychology, 75: 219-229. DOI: 10.1037/00223514.75.1.219

Butler A.C., Hokanson J.E. e Flynn H.A. (1994), “A comparison of selfesteem liability and low trait selfesteem as vulnerability factors in depression", Journal of Personality and Social Psychology, 66: 166-177. DOI: 10.1037/0022-3514.66.1.166

Buunk B.P. (1998), "Social comparison and optimism about one's relational future: Order effects in social judgment", European Journal of Social Psychology, 28: 777-786. DOI: 10.1002/(SICI)1099-0992(199809/10)28:5<777::AID-EJSP892>3.0.CO;2-O

Campbell J.D. (1986), "Similarity and uniqueness: The effects of attribute type, relevance, and individual differences in self-esteem and depression", Journal of Personality and Social Psychology, 50: 281-294. DOI: 10.1037/0022-3514.50.2.281

Canevello A. e Crocker J. (2010), "Creating good relationships: Responsiveness, relationship quality, and interpersonal goals", Journal of Personality and Social Psychology, 99: 78-106. DOI: 10.1037/a0018186

Canevello A. e Crocker J. (2011a), "Interpersonal Goals and Close Relationship Processes: Potential Links to Health", Social and Personality Psychology Compass, 5/6: 346-358. DOI: 10.1111/j.1751-9004.2011.00356.x

Canevello A. e Crocker J. (2011b), “'Interpersonal goals, others' regard for the self, and self-esteem: The paradoxical consequences of self-image and compassionate goals", European Journal of Social Psychology, 41(4): 422-434. DOI: 10.1002/ ejsp. 808

Cantelmi T. e Lambiase E. (2007), "Legame affettivo e comportamento sessuale. Come lo stile di attaccamento influenza il comportamento sessuale", Psicoterapia $e$ Scienze Umane, 41, 2: 205-228.

Carver C.S. e Ganellen R.J. (1983), "Depression and components of selfpunitiveness: High standards, selfcriticism, and overgeneralization", Journal of Abnormal Psychology, 92: 330-337. DOI: 10.1037/0021-843X.92.3.330

Carver C.S. e Scheier M.F. (1998), On the self-regulation of behavior, New York: Cambridge University Press.

Carver C.S. (1998), "Generalization, adverse events, and development of depresssive symptoms", Journal of Personality, 66: 607-619. DOI: 10.1111/1467-6494.00026

Carver C.S. (2003), "Pleasure as a sign you can attend to something else: Placing positive feelings within a general model of affect", Cognition and Emotion, 17: 241261. DOI: 10.1080/02699930302294

Carver C.S., la Voie L., Kuhl J. e Ganellen R.J. (1988), “Cognitive concomitants of depression: A further examination of the roles of generalization, high standards, and self-criticism", Journal of Social and Clinical Psychology, 7: 350-365.

Carver C.S., la Voie L., Kuhl J. e Ganellen R.J. (1988), “Cognitive concomitants of de- 
pression: A further examination of the roles of generalization, high standards, and self-criticism", Journal of Social and Clinical Psychology, 7: 350-365.

Clark M.S. e Lemay E.P.Jr. (2010), Close relationships, in Fiske S.T., Gilbert D.T. e Lindzey G. (eds.), Handbook of Social Psychology (5 ed.), 2: 898-940, New York: Wiley.

Clark M.S., Mills J. e Powell M.C. (1986), "Keeping track of needs in communal and exchange relationships", Journal of Personality and Social Psychology, 51: 333338. DOI: $10.1037 / 0022-3514.51 .2 .333$

Cohen S. e Wills T.A. (1985), "Stress, social support, and the buffering hypothesis", Psychological Bulletin, 98: 310-357.

Cohen S., Gottlieb B.H. e Underwood L.G. (2000), Social relationships and health, New York, NY, US: Oxford University Press.

Cohen S., Sherrod D.R. e Clark M.S. (1986), "Social skills and the stressprotective role of social support", Journal of Personality and Social Psychology, 50: 963-973. DOI: 10.1037/0022-3514.50.5.963

Collins N.L. e Feeney B. (2000), "A safe haven: An attachment theory perspective on support seeking and care giving in close relationships", Journal of Personality and Social Psychology, 78: 1053-1073. DOI: 10.1037/0022-3514.78.6.1053

Colvin C.R. e Block J. (1994), "Do positive illusions foster mental health? An examination of the Taylor and Brown formulation", Psychological Bulletin, 116: 3-20. DOI: $10.1037 / 0033-2909.116 .1 .3$

Cooley C.H. (1956), Human nature and the social order, New York: Schocken. (ed. orig. 1902).

Cooper M.L., Agocha V.B. e Sheldon M.S. (2000), “A motivational perspective on risky behaviors: The role of personality and affect regulatory processes", Journal of Personality: Special Issue: Personality processes and problem behavior, 68: 10591088. DOI: 10.1111/1467-6494.00126

Cooper M.L., Frone M.R., Russell M. e Mudar P. (1995), "Drinking to regulate positive and negative emotions: A motivational model of alcohol use", Journal of Personality and Social Psychology, 69: 990-1005. DOI: 10.1037/0022-3514.69.5.990

Cooper M.L., Shapiro C.M. e Powers A.M. (1998), "Motivations for sex and risky sexual behavior among adolescents and young adults: A functional perspective", Journal of Personality and Social Psychology, 75: 1528-1558. DOI: 10.1037/00223514.75.6.1528

Coopersmith S. (1967), The antecedents of self-esteem, San Francisco: Freeman.

Covington M.V. (1984). "The self-worth theory of achievement motivation: Findings and implications", Elementary School Journal, 85: 520.

Covington M.V. (2000), "Goal theory, motivation, and school achievement: An integrative review”, Annual Review of Psychology, 51: 171-200. DOI: 10.1146/ annurev.psych.51.1.171

Crary W.G. (1966), "Reactions to incongruent self-experiences", Journal of Consulting Psychology, 30: 246-252. DOI: 10.1037/h0023347

Crocker J. e Canevello A. (2008), "Creating and undermining social support in communal relationships: The roles of compassionate and self-image goals", Journal of Personality and Social Psychology, 95: 555-575. DOI: 10.1037/0022-3514.95. 3.555

Crocker J. e Luhtanen R.K. (1990), “Collective self-esteem and ingroup bias”, Journal of Personality and Social Psychology, 58: 60-67. DOI: 10.1037/0022-3514.58.1.60 
Crocker J. e Luhtanen R.K. (2003), "Level of self-esteem and contingencies of selfworth: Unique effects on academic, social, and financial problems in college freshmen", Personality and Social Psychology Bulletin, 29: 701-712. DOI: 10.1177/0146167203029006003

Crocker J. e Nuer N. (2004), "Do People Need Self-Esteem? Comment on Pyszczynski et al. (2004)", Psychological Bulletin, 130, 3: 469-472. DOI: 10.1037/00332909.130.3.469

Crocker J. e Park L.E. (2004), “The costly pursuit of self-esteem”, Psychological Bulletin, 130: 392-414. DOI: 10.1037/0033-2909.130.3.392

Crocker J. e Park L.E. (2003), Seeking self-esteem: Maintenance, enhancement, and protection of selfworth, in Leary M.R. e Tangney J. (eds.), Handbook of self and identity, 291-313, New York: Guilford.

Crocker J. e Wolfe C.T. (2001), “Contingencies of self-worth”, Psychological Review, 108: 593-623. DOI: 10.1037/0033-295X.108.3.593

Crocker J. (1993), "Memory for information about others: Effects of self-esteem and performance feedback", Journal of Research in Personality, 27: 35-48. DOI: 10.1006/jrpe.1993.1004

Crocker J. (2002), “The costs of seeking self-esteem”, Journal of Social Issues, 58: 597-615. DOI: 10.1111/1540-4560.00279

Crocker J., Canevello A., Breines J. e Flynn H. (2010), "Interpersonal goals and change in anxiety and dysphoria in first-semester college students", Journal of Personality and Social Psychology, 98, 1009-1024. DOI: 10.1037/a0019400

Crocker J., Karpinski A., Quinn D.M. e Chase S. (2003), "When grades determine selfworth: Consequences of contingent self-worth for male and female engineering and psychology majors", Journal of Personality and Social Psychology, 85(3): 507-516. DOI: 10.1037/0022-3514.85.3.507

Crocker J., Sommers S.R. e Luhtanen R.K. (2002), "Hopes dashed and dreams fulfilled: Contingencies of self-worth and admissions to graduate school", Personality and Social Psychology Bulletin, 28: 1275-1286. DOI: 10.1177/01461672022812012

Crocker J., Thompson L., McGraw K. e Ingerman C. (1987), "Downward comparison, prejudice, and evaluation of others: Effects of selfesteem and threat", Journal of Personality and Social Psychology, 52: 907-916. DOI: 10.1037/0022-3514.52. 5.907

Damon W. (1995), Greater expectations: Overcoming the culture of indulgence in America's homes and schools, New York: Free Press.

Davis M.H. (1983), "Measuring individual differences in empathy: Evidence for a multidimensional approach", Journal of Personality and Social Psychology, 44: 113126. DOI: $10.1037 / 0022-3514.44 .1 .113$

Dawes R.M. (1994), House of cards: Psychology and psychotherapy built on myth, New York: Free Press.

deCharms R. (1968). Personal causation, New York: Academic Press.

Deci E.L. e Ryan R.M. (1995), Human autonomy: The basis for true self-esteem, in Kernis M.H. (ed.), Efficacy, agency, and self-esteem, 31-49. New York: Plenum.

Deci E.L. e Ryan R.M. (2000), “The 'what' and 'why' of goal pursuits: Human needs and the selfdetermination of behavior", Psychological Inquiry, 11: 227-268. DOI: 10.1207/S15327965PLI1104_01

Deci E.L., Eghrari H., Patrick B.C. e Leone D.R. (1994), "Facilitating internalization: The selfdetermination theory perspective", Journal of Personality, 62: 119-142. DOI: 10.1111/j.1467-6494.1994.tb00797.x 
Deci E.L., La Guardia J.G., Moller A.C., Scheiner M.J. e Ryan R.M. (2006), “On the benefits of giving as well as receiving autonomy support: Mutuality in close friendships", Personality and Social Psychology Bulletin, 32: 313-327. DOI: $10.1177 / 0146167205282148$

Deci E.L., Nezlek J. e Sheinman L. (1981), "Characteristics of the rewarder and intrinsic motivation of the rewardee", Journal of Personality and Social Psychology, 40: 1-10. DOI: $10.1037 / 0022-3514.40 .1 .1$

Dickerson S.S. e Kemeny M.E. (2004), “Acute stressors and cortisol responses: A theoretical integration and synthesis of laboratory research", Psychological Bulletin, 130: 355-391. DOI: 10.1037/0033-2909.130.3.355

Downey G. e Feldman S. (1996), "Implications of rejection sensitivity for intimate relationships", Journal of Personality and Social Psychology, 70: 1327-1343. DOI: 10.1037/0022-3514.70.6.1327

Downey G., Freitas A.L., Michaelis B. e Khouri H. (1998), "The self-fulfilling prophecy in close relationships: Rejection sensitivity and rejection by romantic partners", Journal of Personality and Social Psychology, 75: 545-560. DOI: 10.1037/00223514.75.2.545

Dunn E.W., Aknin L.B. e Norton M.I. (2008), "Spending money on others promotes happiness", Science, 319: 1687-1688. DOI: 10.1126/science.1150952

Dweck C.S. (2000), Self-theories: Their role in motivation, personality, and development, Philadelphia: Psychology Press.

Dweck C.S., Chiu C. e Hong Y. (1995), "Implicit theories and their role in judgements and reactions: A world from two perspectives", Psychological Inquiry, 6(4): 267285. DOI: $10.1207 / \mathrm{s} 15327965$ pli0604_1

Dykman B.M. (1998), "Integrating cognitive and motivational factors in depresssion: Initial tests of a goalorientation approach", Journal of Personality and Social Psychology, 74: 139-158. DOI: 10.1037/0022-3514.74.1.139

Ellis A. e London T. (1993), The case against self-esteem: How the self-esteem movement is damaging our children and culture, Chicago: Garfield Press.

Enright R.D., Freedman S. e Rique J. (1998), The psychology of interpersonal forgiveness, in Enright R.D. e North J. (eds.), Exploring forgiveness, 46-62, Madison, WI: University of Wisconsin Press.

Feather N.T. (1994), Attitudes toward high achievers and reactions to their fall: Theory and research concerning tall poppies, in Zanna M. (ed.), Advances in experimental social psychology, 1-73, San Diego, CA: Academic Press.

Feeney B.C. e Collins N.L. (2003), "Motivations for caregiving in adult intimate relationships: Influences on caregiving behavior and relationship functioning", Personality and Social Psychology Bulletin, 29: 950-968. DOI: 10.1177/ 0146167203252807

Fehr B. e Sprecher S. (2009), "Prototype analysis of the concept of compassionate love", Personal Relationships, 16: 343-364. DOI: 10.1111/j.1475-6811.2009. 01227.x

Fein S. e Spencer S.J. (1997), "Prejudice as self-image maintenance: Affirming the self through derogating others", Journal of Personality and Social Psychology, 73: 3144. DOI: $10.1037 / 0022-3514.73 .1 .31$

Finn C.E. (1990), "Narcissus goes to school”, Commentary, 89: 40-45.

Franks D.D. e Marolla J. (1976), "Efficacious action and social approval as interacting dimensions of self-esteem: A tentative formulation through construct validation", Sociometry, 39: 324-341. DOI: 10.2307/3033498 
Fredrickson B.L. e Branigan C. (2001), Positive emotions, in Bonanno T.J.M.G.A. (ed.), Emotions: Current issues and future directions, 123-151, New York: Guilford.

Gable S.L. e Reis H.T. (2006), Intimacy and the self: An iterative model of the self and close relationships, in Noller P. e Feeney J.A. (eds.), Close Relationships: Functions, Forms and Processes, 211-225, Hove, England: Psychology Press / Taylor and Francis (UK).

Gecas V. e Schwalbe M.L. (1983), "Beyond the looking-glass self: Social structure and efficacy-based selfesteem", Social Psychology Quarterly, 46: 77-88.

Gilbert P. e Irons C. (2005), Therapies for shame and self-attacking, using cognitive, behavioural, emotional imagery and compassionate mind training, in Gilbert P. (Ed), Compassion: Conceptualisations, research and use in psychotherapy, 263325, London: Routledge.

Glennon W. (1999), 200 Ways to Raise a Girl's Self-Esteem: An Indespensable Guide for Parents, Teachers and Other Concerned Caregivers, Newburyport, MA: Conari Press.

Goldstein A.P. e Michaels G.Y. (1985), Empathy: Development, training, and consequences. Hillsdale, NJ: Erlbaum.

Goldstein J. e Kornfield J. (1987), Seeking the heart of wisdom: The path of insight meditation. Boston: Shambhala.

Goleman D. (1995) Emotional Intelligence, New York, NY, England: Bantam Books, Inc.

Gollwitzer P.M. e Wicklund R. (1985), "Self-symbolizing and the neglect of others' perspectives", Journal of Personality and Social Psychology, 43: 702-715. DOI: 10.1037/0022-3514.48.3.702

Greenberg J. e Pyszczynski T. (1985), "Compensatory self-inflation: A response to the threat to self-regard of public failure", Journal of Personality and Social Psychology, 49: 273-280. DOI: 10.1037/0022-3514.49.1.273

Haidt J. (2003), The moral emotion, in Davidson R.J., Scherer K.R. e Goldsmith H.H. (eds.), Handbook of affective sciences, 852-870, Oxford: Oxford University Press.

Harter S. (1999), The Construction of the self: A developmental perspective. New York, NY: Guilford Press.

Hawkley L.C. e Cacioppo J.T. (2003), "Loneliness and pathways to disease", Brain, Behavior, and Immunity, 17: S98-S105. DOI: 10.1016/S0889-1591(02)00073-9

Heatherton T.F. e Vohs K.D. (2000), "Interpersonal evaluations following threat to self", Journal of Personality and Social Psychology, 78: 725-736. DOI: 10.1037/0022-3514.78.4.725

Heine S.J., Lehman D.R., Markus H.R. e Kitayama S. (1999), "Is there a universal need for positive self-regard?", Psychological Review, 106: 766-794.

Helgeson V.S. (1994), "Relation of agency and communion to well-being: Evidence and potential explanations", Psychological Bulletin, 116: 412-428.

Hellhammer D.H. e Wade S. (1993), "Endocrine correlates of stress vulnerability", Psychother Psychosom, 60: 8-17. DOI: 10.1159/000288675

Henry J.P. e Wang S. (1998), "Effects of early stress on adult affiliative behavior", Psychoneuroendocrinology, 23: 863-875. DOI: 10.1016/S0306-4530(98)00058-4

Hewitt J.P. (1998), The myth of self-esteem: Finding happiness and solving problems in America, New York: St. Martin's Press.

Horney K. (1950), Neurosis and human growth: The struggle toward self-realization, New York: Norton. 
House J.S., Landis K.R. e Umberson D. (1988), "Social relationships and health", Science, 241: 540-545.

Hull J.G. e Young R.D. (1983), "Selfconsciousness, self-esteem, and success-failure as determinants of alcohol consumption in male social drinkers", Journal of Personality and Social Psychology, 44: 1097-1109. DOI: 10.1037/0022-3514.44.6.1097

Hull J.G. (1981), "A self-awareness model of the causes and effects of alcohol consumption”, Journal of Abnormal Psychology, 90: 586-600. DOI: 10.1037/0021$843 X .90 .6 .586$

Hull J.G., Levenson R.W., Young R.D. e Sher K.J. (1983), "Self-awareness reducing effects of alcohol consumption", Journal of Personality and Social Psychology, 44: 461-473. DOI: 10.1037/0022-3514.44.3.461

James W. (1890), Theprinciplesofpsychology, 1, Cambridge, MA: Harvard University Press.

Johnson E.A. e O'Brien K.A. (2013), “Self-compassion soothes the savage ego-threat system: effects on negative affect, shame, rumination, and depressive symptoms", Journal of Social and Clinical Psychology, 32(9): 939-963.

Joiner T.E. (1994), “Contagious depression: Existence, specificity to depressed symptoms, and the role of reassurance seeking", Journal of Personality and Social Psychology, 67: 287-296. DOI: 10.1037/0022-3514.67.2.287

Joiner T.E., Alfano M.S. e Metalsky G.I. (1992), "When depression breeds contempt: Reassurance seeking, selfesteem, and rejection of depressed college students by their roommates", Journal of Abnormal Psychology, 101: 165-173. DOI: 10.1037/0021-843X.101.1.165

Joiner T.E., Katz J. e Lew A. (1999), "Harbingers of depressotypic reassurance seeking: Negative life events, increased anxiety, and decreased self-esteem", Personality and Social Psychology Bulletin, 25: 632-639. DOI: 10.1177/0146167299025005008

Joiner T.E., Metalsky G.I., Katz J. e Beach S.R.H. (1999), "Depression and excessive reassurance-seeking", Psychological Inquiry, 10: 269-278. DOI: 10.1207/ S15327965PLI1004_1

Jones E.E. e Pittman T.S. (1982), Toward a general theory of strategic selfpresentation, in Suls J. (ed.), Psychological Perspectives on the Self, 231-262, Hillsdale, NJ: Erlbaum.

Kelly A.C., Zuroff D.C. e Shapira L.B. (2009), "Soothing oneself and resisting selfattacks: The treatment of two intrapersonal deficits in depression vulnerability", Cognitive Therapy and Research, 33: 301-313. DOI: 10.1007/s10608-008-9202-1

Kenny D.A. e Acitelli L.K. (2001), "Accuracy and bias in the perception of the partner in a close relationship", Journal of Personality and Social Psychology, 80, 3: 439448. DOI: 10.1037/0022-3514.80.3.439

Kernis M.H. e Waschull S.B. (1995), The interactive roles of stability and level of selfesteem: Research and theory, in Zanna M.P. (ed.), Advances in experimental social psychology, 27: 93-141, San Diego, CA: Academic Press.

Kernis M.H. (2003), “Toward a conceptualization of optimal selfesteem”, Psychological Inquiry, 14: 1-26. DOI: 10.1207/S15327965PLI1401_01

Kernis M.H., Whisenhunt C.R., Waschull S.B., Greenier K.D., Berry A.J., Herlocker C.E. e Anderson C.A. (1998), "Multiple facets of selfesteem and their relations to depressive symptoms", Personality and Social Psychology Bulletin, 24: 657-668. DOI: $10.1177 / 0146167298246009$ 
Kiecolt-Glaser J. e Glaser R. (1994), “The effects of an acute psychological stressor on cardiovascular, endocrine, and cellular immune response: A prospective study of individuals high and low in heart rate reactivity", Psychophysiology, 31: 264-271. DOI: 10.1111/j.1469-8986.1994.tb02215.x

Kiecolt-Glaser J., Cacioppo J., Malarkey W. e Glaser R. (1992), “Acute psychological stressors and shortterm immune changes: What, why, for whom and to what extent?", Psychosomatic Medicine, 53: 345-362.

Kiecolt-Glaser J.K., Bane C., Glaser R. e Malarkey W.B. (2003), "Love, marriage, and divorce: Newlyweds' stress hormones foreshadow relationship changes", Journal of Consulting and Clinical Psychology, 71: 176-188. DOI: 10.1037/0022006X.71.1.176

Krause N. e Shaw B.A. (2000), "Giving social support to others, socioeconomic status, and changes in self-esteem in late life", Journals of Gerontology. Series B, Psychological Sciences and Social Sciences, 55b: S323-S333. DOI: 10.1093/geronb/ 55.6.S323

Krause N.M., Herzog A.R. e Baker E. (1992), "Providing support to others and wellbeing in later life", Journals of Gerontology, 47: 300-311. DOI: 10.1093/geronj/ 47.5.P300

Kuiper N.A. e Olinger L.J. (1986), Dysfunctional attitudes and a selfworth contingency model of depression, in Kendall P.C. (ed.), Advances in cognitive-behavioral research and therapy, 115-142, Orlando, FL: Academic Press.

Kuiper N.A., Olinger L.J. e MacDonald M.R. (1988), Vulnerability and episodic cognitions in a self-worth contingency model of depression, in Alloy L.B. (ed.), Cognitive processes in depression, 289-309, New York: Guilford Press.

Leary M.R. e Baumeister R.F. (2000), The nature and function of selfesteem: Sociometer theory, in Zanna M. (ed.), Advances in Experimental Social Psychology, 32: 162, San Diego, CA: Academic Press.

Leary M.R. e Kowalski R.M. (1990), "Impression management: A literature review and two-component model”, Psychological Bulletin, 107: 34-47. DOI: 10.1037/00332909.107.1.34

Leary M.R. (1995), Self-Presentation: Impression Management and Interpersonal Behavior. Madison, WI: Brown and Benchmark Publishers.

Leary M.R. (2004), The curse of the self: Self-awareness, egotism, and the quality of human life. New York: Oxford University Press.

Leary M.R. (2007), "Motivational and emotional aspects of the self”, Annual Review of Psychology, 58: 317-344. DOI: 10.1146/annurev.psych.58.110405. 085658

Leary M.R., Tate E.B., Adams C.E., Allen A.B. e Hancock J. (2007), "Self-compassion and reactions to unpleasant self-relevant events: The implications of treating oneself kindly", Journal of Personality and Social Psychology, 92: 887-904. DOI: 10.1037/00223514.92.5.887

Lemay E.P. Jr. e Clark M.S. (2008), "How the head liberates the heart: Projection of communal responsiveness guides relationship promotion", Journal of Personality and Social Psychology, 94: 647-671. DOI: 10.1037/0022-3514.94.4.647

Lemay E.P. Jr., Clark M.S. e Feeney B.C. (2007), "Projection of responsiveness to needs and the construction of satisfying communal relationships", Journal of Personality and Social Psychology, 92: 834-853. DOI: 10.1037/0022-3514.92.5.834

Lewis M. (1993), Self-conscious emotions: Embarrassment, pride, shame, and guilt, in Haviland M.L.J.M. (ed.), Handbook of emotions, 563-573, New York: Guilford. 
Marshall S.L., Parker P.D., Ciarrochi J., Sahdra B. e Heaven P.C.L. (2015), “Selfcompassion protects against the negative effects of low self-esteem: A longitudinal study in a large adolescent sample", Personality and Individual Differences, 74: 116-121. DOI: 10.1016/j.paid.2014.09.013

Mascolo M.F. e Fischer K.W. (1995), Developmental transformations in appraisals for pride, shame, and guilt, in Fischer J.M.T.K.W. (ed.), Self-conscious emotions: The psychology of shame, guild, embarrassment, and pride, New York: Guilford.

Maslow A.H. (1968), Motivation and personality, New York: Harper and Row.

McElherner L.N. e Lisovskis M. (1998), Jumpstarters: Quick Classroom Activities That Develop Self-Esteem, Creativity, and Cooperation, Golden Valley, MN: Free Spirit Publishing.

McMillan J.H., Singh J. e Simonetta L.G. (1994), "The tyranny of self-oriented selfesteem", Educational Horizons, Sp: 141-145.

Mead G.H. (1934), Mind, self, and society, Chicago: University of Chicago Press.

Mecca A.M., Smelser N.J. e Vasconcellos J. (1989), The social importance of selfesteem, Berkeley, CA: University of California Press.

Metcalfe J. e Mischel W. (1999), “A hot/cool system analysis of delay of gratifycation: Dynamics of willpower", Psychological Review, 106: 3-19. DOI: 10.1037/0033295X.106.1.3

Mikulincer M. e Shaver P.R. (2005), “Attachment security, compassion, and altruism", Current Directions in Psychological Science, 14: 34-38. DOI: 10.1111/j.09637214.2005.00330.x

Millar M.G., Millar K.U. e Tesser A. (1988), "The effects of helping and focus of attention on mood states", Personality and Social Psychology Bulletin, 14: 536-543. DOI: $10.1177 / 0146167288143012$

Miller D.T. e Ross M. (1975), "Selfserving biases in attribution of causality: Fact or fiction?", Psychological Bulletin, 82: 213-225. DOI: http://dx.doi.org/ $10.1037 / \mathrm{h} 0076486$

Miller P.J. (2001), Self-esteem as folk theory: A comparison of ethnographic interviews. Paper presented at the Society for Research in Child Development, Minneapolis, MN.

Mischel W. e Shoda Y. (1995), “A Cognitive-affective system theory of personality: Reconceptualizing situations, dispositions, dynamics, and invariance in personality structure", Psychological Review, 102: 246-268. DOI: 10.1037/0033-295X.102. 2.246

Mischel W., Ebbesen E.B. e Zeiss A.R. (1976), "Determinants of selective memory about the self", Journal of Consulting and Clinical Psychology, 44: 92-103. DOI: 10.1037/0022-006X.44.1.92

Mueller C.M. e Dweck C.S. (1998), "Praise for intelligence can undermine children's motivation and performance", Journal of Personality and Social Psychology, 75(1): 33-52. DOI: 10.1037/0022-3514.75.1.33

Murray S.L., Bellavia G., Feeney B., Holmes J.G. e Rose P. (2001), “The contingencies of interpersonal acceptance: When romantic relationships function as a selfaffirmational resource", Motivation and Emotion, 25: 163-189. DOI: 10.1023/A:1010618010115

Murray S.L., Holmes J.G. e Collins N.L. (2006), “Optimizing assurance: The risk regulation system in relationships", Psychological Bulletin, 132: 641-666. DOI: 10.1037/0033-2909.132.5.641

Murray S.L., Holmes J.G. e Griffin D.W. (1996), “The benefits of positive illusions: 
Idealization and the construction of satisfaction in close relationships", Journal of Personality and Social Psychology, 70: 79-98. DOI: 10.1037/0022-3514.70.1.79

Murray S.L., Holmes J.G. e Griffin D.W. (2000), "Self-esteem and the quest for felt security: How perceived regard regulates attachment processes", Journal of Personality and Social Psychology, 78: 478-498. DOI: 10.1037/0022-3514. 78.3.478

Murray S.L., Holmes J.G., Griffin D.W., Bellavia G. e Rose P. (2001), "The mismeasure of love: How self-doubt contaminates relationship beliefs", Personality and Social Psychology Bulletin, 27: 423-436. DOI: 10.1177/ 0146167201274004

Musick M.A., Herzog A.R. e House J.S. (1999), "Volunteering and mortality among older adults: Findings from a national sample", Journal of Gerontology: Social Sciences, 54b: S173-S180. DOI: 10.1093/geronb/54B.3.S173

Neff K.D. e McGeehee P. (2010), "Self-compassion and psychological resilience among adolescents and young adults", Self and Identity, 9: 225-240. DOI: $10.1080 / 15298860902979307$

Neff K.D. e Vonk R. (2009), "Self-Compassion Versus Global Self-Esteem: Two Different Ways of Relating to Oneself", Journal of Personality, 77, 1: 23-50. DOI: $10.1111 / \mathrm{j} .1467-6494.2008 .00537 . \mathrm{x}$

Neff K.D. (2003a), "The development and validation of a scale to measure selfcompassion", Self and Identity, 2: 223-250. DOI: 10.1080/15298860309027

Neff K.D. (2003b), "Self-Compassion: An Alternative Conceptualization of a Healthy Attitude Toward Oneself", Self and Identity, 2: 85-101. DOI: 10.1080/ 15298860309032

Neff K.D. (2006), The role of self-compassion in healthy relationship interactions. Paper presented at the 114th annual meeting of the American Psychological Association, New Orleans, LA.

Neff K.D. (2009), Self-compassion, in Leary M.R. e Hoyle R.H. (eds.), Handbook of individual differences in social behavior, 561-573, New York, NY: Guilford Press.

Neff K.D. (2011), Self-Compassion. New York: HarperCollins.

Neff K.D., Hseih Y. e Dejitthirat K. (2005), "Self-compassion, achievement goals, and coping with academic failure", Self and Identity, 4: 263-287. DOI: 10.1080/ 13576500444000317

Neff K.D., Hseih Y. e Dejitthirat K. (2005), "Self-compassion, achievement goals, and coping with academic failure", Self and Identity, 4: 263-287. DOI: 10.1080/ 13576500444000317

Neff K.D., Kirkpatrick K. e Rude S.S. (2007), "Self-compassion and its link to adaptive psychological functioning", Journal of Research in Personality, 41: 139-154. DOI: 10.1016/j.jrp.2006.03.004

Neff K.D., Pisitsungkagarn K. e Hseih Y. (2008), "Self-compassion and self-construal in the United States, Thailand, and Taiwan", Journal of Cross-Cultural Psychology, 39: 267-285. DOI: 10.1177/0022022108314544

Neff K.D., Rude S.S. e Kirkpatrick K. (2007), “An examination of self-compassion in relation to positive psychological functioning and personality traits", Journal of Research in Personality, 41: 908-916. DOI: 10.1016/j.jrp.2006.08.002

Nolen-Hoeksema S. (1991), "Responses to depression and their effects on the duration of depressive episodes", Journal of Abnormal Psychology, 100: 569-582. DOI: 10.1037/0021-843X.100.4.569

Oman D., Thoresen C.E. e McMahon K. (1999), "Volunteerism and mortality among 
the community-dwelling elderly", Health Psychology, 4: 301-316. DOI: 10.1177/ 135910539900400301

Park L.E. e Crocker J. (2005), “The interpersonal costs of seeking selfesteem”, Personality and Sociale Psychology Bullettin, 31, 11: 1587-1598. DOI: 10.1177/ 0146167205277206

Piferi R.L. e Lawler K.A. (2006), "Social support and ambulatory blood pressure: An examination of both receiving and giving", International Journal of Psychophysiology, 62: 328-336. DOI: 10.1016/j.ijpsycho.2006.06.002

Post S.G. (ed.) (2007), Altruism and Health: Perspectives from Empirical Research, New York, NY: Oxford University Press.

Pyszczynski T. e Greenberg J. (1987), "Self-regulatory perseveration and the depressive self-focusing style: A self-awareness theory of reactive depression", Psychological Bulletin, 102: 122-138.

Pyszczynski T., Greenberg J. e Goldenberg J. (2002), Freedom in the Balance: On the Defense, Growth, and Expansion of the Self, in Leary M. e Tangney J. (eds.), Handbook of self and identity (314-343), New York: Guilford.

Pyszczynski T., Greenberg J. e Laprelle J. (1985), "Social comparison after success and failure: Biased search for information consistent with a self-serving conclusion", Journal of Experimental Social Psychology, 21: 195-211. DOI: 10.1016/00221031(85)90015-0

Pyszczynski T., Greenberg J. e Solomon S. (1997), "Why do we need what we need? A terror management perspective on the roots of human social motivation", Psychological Inquiry, 8: 1-20. DOI: 10.1207/s15327965pli0801_1

Raskin R. e Hall C.S. (1979), “A Narcissistic Personality Inventory”, Psychological Reports, 45: 590.

Reich W. (1949), Character analysis, New York: Orgone Institute Press.

Reis H.T., Clark M.S. e Holmes J.G. (2004), Perceived partner responsiveness as an organizing construct in the study of intimacy and closeness, in Mashek D.J. e Aron A.P. (eds.), Handbook of Closeness and Intimacy, 201-225, Mahwah, NJ: Lawrence Erlbaum Associates Publishers.

Reis H.T., Sheldon K.M., Gable S.L., Roscoe J. e Ryan R.M. (2000), "Daily wellbeing: The role of autonomy, competence, and relatedness", Personality and Social Psychology Bulletin, 26: 419-435. DOI: 10.1177/0146167200266002

Roberts J.E. e Gamble S.A. (2001), "Current mood-state and past depression as predictors of selfesteem and dysfunctional attitudes among adolescents", Personality and Individual Differences, 30: 1023-1037. DOI: 10.1016/S01918869(00)00093-3

Roberts J.E. e Gotlib I.H. (1997), "Temporal variability in global selfesteem and specific self-evaluation as prospective predictors of emotional distress: Specificity in predictors and outcome", Journal of Abnormal Psychology, 106: 521-529. DOI: 10.1037/0021843X.106.4.521

Roberts J.E. e Monroe S.M. (1992), "Vulnerable self-esteem and depressive symptoms: Prospective findings comparing three alternative conceptualizations", Journal of Personality and Social Psychology, 62: 804-812. DOI: 10.1037/0022-3514.62.5.804

Robins R.W. e Beer J.S. (2001), "Positive illusions about the self: Short-term benefits and long-term costs", Journal of Personality and Social Psychology, 80: 340-352. DOI: $10.1037 / 0022-3514.80 .2 .340$

Rogers C.R. (1961), On becoming a person, Boston: Houghton Mifflin. 
Rosenberg M. (1965), Society and the adolescent self-image, Princeton, NJ: Princeton University Press.

Rosenberg M. (1979), Conceiving the self, New York: Basic Books.

Ryan R.M. e Connell J.P. (1989), "Perceived locus of causality and internalization: Examining reasons for acting in two domains", Journal of Personality and Social Psychology, 57: 749-761. DOI: 10.1037/0022-3514.57.5.749

Ryan R.M. (1982), "Control and information in the interpersonal sphere: An extension of cognitive evaluation theory", Journal of Personality and Social Psychology, 43: 450-461. DOI: 10.1037/0022-3514.43.3.450

Ryff C.D. (1995), "Psychological well-being in adult life", Psychological Science, 4: 99-104.

Sapolsky R.M (1998), Why zebras don't get ulcers: An updated guide to stress, stressrelated diseases, and coping, New York: Freeman.

Scheff T.J. (1981), "The distancing of emotion in psychotherapy", Psychotherapy: Theory, Research e Practice, 18: 46-53. DOI: 10.1037/h0085960

Schimel J., Arndt J., Pyszczynski T. e Greenberg J. (2001), "Being accepted for who we are: evidence that social validation of the intrinsic self reduces general defensiveness", Journal of Personality and Social Psychology, 80, 1: 35-52. DOI: 10.1037/0022-3514.80.1.35

Schlenker B.R. (1980), Impression Management: The Self-Concept, Social Identity, and Interpersonal Relations, Monterey, CA: Brooks/Cole.

Schlenker B.R. (2003), Self-presentation, in Leary M.R. e Tangney J.P. (eds.), Handbook of self and identity, 492-518, New York: Guilford Press.

Schwartz C., Meisenhelder J.B., Ma Y. e Reed G. (2003), “Altruistic social interest behaviors are associated with better mental health", Psychosomatic Medicine, 65: 778-785.

Seligman M.E.P. (1995), The optimistic child, Boston: Houghton Mifflin.

Seligman M.E.P. (1998), “The American way of blame”, APA Monitor, 29: 4.

Sheldon K.M., Elliot A. J., Kim Y. e Kasser T. (2001), "What is satisfying about satisfying events? Testing candidate psychological needs", Journal of Personality and Social Psychology, 80: 325-339. DOI: 10.1037/0022-3514.80.2.325

Shoda Y., Mischel W. e Peake P.K. (1990), "Predicting adolescent cognitive and selfregulatory competencies from preschool delay of gratification: Idntifying diagnostic conditions", Developmental Psychology, 26: 978-986. DOI: 10.1037/0012-1649.26.6.978

Solomon S., Greenberg J. e Pyszczynski T. (1991), A terror-management theory of social behavior: The psychological functions of selfesteem and cultural worldviews, in Zanna M.P. (ed.), Advances in experimental social psychology, 24: 91-159, San Diego, CA: Academic Press.

Spencer S.J., Fein S., Wolfe C.T., Fong C. e Dunn M.A. (1998), “Automatic activation of stereotypes: The role of self-image threat", Personality and Social Psychology Bulletin, 24: 1139-1152. DOI: 10.1177/01461672982411001

Steele C.M., Spencer S.J. e Aronson J. (2002), Contending with group image: The psychology of stereotype and social identity threat, in Zanna M.P. (ed.) Advances in Experimental Social Psychology, 34: 379-440, San Diego: Academic Press.

Stroebe W. (2000), Health Psychology (2nd ed.), Buckingham, England: Open University Press.

Suinn R.M. (2001), "The terrible twos: Anger and anxiety", American Psychologist, 56: 27-36. DOI: 10.1037/0003-066X.56.1.27 
Suls J. e Wills T.A. (1991), Social comparison: Contemporary theory and research, Hillsdale, NJ: Erlbaum.

Swann W.B. (1996), Self-traps: The elusive quest for higher self-esteem, New York: Freeman.

Tafarodi R.W. e Swann W.B. Jr. (2001), “Two-dimensional selfesteem: Theory and measurement”, Personality and Individual Differences, Special Issue, 31: 653-673. DOI: $10.1016 / \mathrm{S} 0191-8869(00) 00169-0$

Tangney J.P. (1999), "The self-conscious emotions: Shame, guilt, embarrassment and pride", Handbook of cognition and emotion (541-568), Chichester, England: John Wiley e Sons Ltd.

Taylor J. e Turner R.J. (2001), “A longitudinal study of the role and significance of mattering to others for depressive symptoms", Journal of Health and Social Behavior, 42: 310-325.

Taylor S.E. e Brown J.D. (1988), "Illusion and well-being: A social psychological perspective on mental health", Psychological Bulletin, 103: 193-210. DOI: 10.1037/0033-2909.103.2.193

Taylor S.E., Klein L.C., Lewis B.P., Gruenewald T.L., Gurung R.A.R. e Updegraff J.A. (2000), "Biobehavioral responses to stress in females: Tend-and-befriend, not fightor-flight", Psychological Review, 197: 411-429.

Tedeschi J.T. (1981), Impression Management Theory and Social Psychological Research, New York: Academic.

Tennen H. e Herzberger S. (1987), "Depression, self-esteem, and the absence of selfprotective attributional biases", Journal of Personality and Social Psychology, 52: 72-80. DOI: 10.1037/0022-3514.52.1.72

Tesser A. (1988), Toward a self-evaluation maintenance model of social behavior, in Berkowitz L. (ed.), Advances in experimental social psychology, 21: 181-227, San Diego, CA: Academic Press.

Tesser A. (2000). "On the confluence of self-esteem maintenance mechanisms", Personality and Social Psychology Review, 4: 290-299. DOI: 10.1207/ S15327957PSPR0404_1

Thoits P.A. e Hewitt L.N. (2001), "Volunteer work and well-being", Journal of Health and Social Behavior, 42: 115-131.

Tice D.M. e Bratslavsky E. (2000), "Giving in to feel good: The place of emotion regulation in the context of general self-control", Psychological Inquiry, 11: 149-159. DOI: 10.1207/S15327965PLI1103 03

Tice D.M. e Baumeister R.F. (1997), "Longitudinal study of procrastination, performance, stress, and health: The costs and benefits of dawdling", Psychological Science, 8: 454-458.

Tice D.M. (1991), "Esteem protection or enhancement? Self-handicapping motives and attributions differ by trait self-esteem", Journal of Personality and Social Psychology, 60: 711-725.

Tice D.M. (1993), The social motivations of people with low self-esteem, in Baumeister R. (ed.), Self-esteem: The puzzle of low self-regard, 37-54. Hillsdale, NJ: Erlbaum.

Tice D.M., Bratslavsky E. e Baumeister R.F. (2001), "Emotional distress regulation takes precedence over impulse control: If you feel bad, do it!", Journal of Personality and Social Psychology, 80: 53-67.

Turner J.C., Hogg M.A., Oakes P.J., Reicher S.D. e Wetherell M.S. (1987), Rediscovering the social group: A self-categorization theory, Oxford, UK: Basil Blackwell. 
Twenge J.M., Baumeister R.F., Tice D.M. e Stucke T.S. (2001), "If you can't join them, beat them: Effects of social exclusion on aggressive behavior", Journal of Personality and Social Psychology, 81: 1058-1069.

Twenge J.M., Catanese K.R. e Baumeister R.F. (2002), "Social exclusion causes self- defeating behavior", Journal of Personality and Social Psychology, 83: 606-615.

Uchino B.N. (2004), Social Support and Physical Health: Understanding the Health Consequences of Relationships, New Haven, CT: Yale University Press.

Vohs K.D. e Heatherton T.F. (2000), "Self-regulatory failure: A resourcedepletion approach", Psychological Science, Special Issue, 11: 249-254. DOI: 10.1111/14679280.00250

Vohs K.D. e Heatherton T.F. (2001), "Self-esteem and threats to self: Implications for self-construals and interpersonal percep tions", Journal of Personality and Social Psychology, 81: 1103-1118.

White R.W. (1959), "Motivation reconsidered: The concept of competence", Psychological Review, 66: 297-333.

Wills T.A. (1981), "Downward comparison principles in social psychology", Psychological Bulletin, 90: 245-271.

Wilson A.E. e Ross M. (2000), "The frequency of temporal-self and social comparisons in people's personal appraisals", Journal of Personality and Social Psychology, 78: 928-942.

Wilson A.E. e Ross M. (2001), "From chump to champ: People's appraisals of their earlier and present selves", Journal of Personality and Social Psychology, 80: 572-584.

Wispe L. (1991), The psychology of sympathy, New York: Plenum.

Wood J.V., Giordano-Beech M. e Ducharme M.J. (1999), “Compensating for failure through social comparison”, Personality and Social Psychology Bulletin, 25: 13701386. DOI: $10.1177 / 0146167299259004$

Wood J.V., Saltzberg J.A., Neale J.M. e Stone A. (1990), "Self-focused attention, coping responses, and distressed mood in everyday life", Journal of Personality e Social Psychology, 58: 1027-1036.

Yarnell L.M. e Neff K.D. (2013), "Self-compassion, Interpersonal Conflict Resolutions, and Well-being”, Self and Identity, 12: 146-159. DOI: 10.1080/15298868.2011. 649545

Yogev A. e Ronen R. (1982), “Cross-age tutoring: Effects on tutors' attributes”, Journal of Educational Research, 75: 261-268. DOI: 10.1080/00220671.1982. 10885392 